Revue des patrimoines

Le cheval dans le transport public au XIXe siècle, à travers les collections du musée national de la Voiture et du Tourisme, Compiègne

\title{
Élise Fau
}

\section{(2) OpenEdition}

\section{Journals}

Édition électronique

URL : http://journals.openedition.org/insitu/12124

DOI : 10.4000/insitu. 12124

ISSN : 1630-7305

Éditeur

Ministère de la culture

Référence électronique

Élise Fau, «Le cheval dans le transport public au XIXe siècle, à travers les collections du musée national de la Voiture et du Tourisme, Compiègne », In Situ [En ligne], 27 | 2015, mis en ligne le 26 octobre 2015, consulté le 19 avril 2019. URL : http://journals.openedition.org/insitu/12124; DOI : 10.4000/insitu. 12124

Ce document a été généré automatiquement le 19 avril 2019

\section{(c) (i) ()}

In Situ Revues des patrimoines est mis à disposition selon les termes de la licence Creative Commons Attribution - Pas d'Utilisation Commerciale - Pas de Modification 4.0 International. 


\title{
Le cheval dans le transport public au XIXe siècle, à travers les collections $d u$ musée national de la Voiture et du Tourisme, Compiègne
}

\author{
Élise Fau
}

\section{Introduction}

1 Cet article présente un florilège de représentations montrant le cheval dans le transport public au xix ${ }^{\mathrm{e}}$ siècle provenant du fonds d'arts graphiques et de photographies ainsi que de la collection de peintures du musée de la Voiture et du Tourisme de Compiègne.

2 À travers cette sélection, nous avons souhaité tout d'abord rassembler un échantillon, qui, sans être exhaustif, soit suffisamment représentatif des principaux types de voitures publiques caractérisant l'apogée d'un système de transport fondé sur le cheval. Celles-ci, telles les fiacres, omnibus ou diligences, sont aujourd'hui encore investies d'une indéniable aura imaginaire, bien que leurs caractéristiques soient assez largement méconnues. Nous chercherons à mettre en évidence les spécificités des différents types de voitures et d'attelages représentés. L'observation s'attachera en particulier aux différents moments du travail des chevaux, à la représentation de leur posture et de leur allure, de leur effort, ainsi qu'aux difficultés, aléas ou accidents qu'ils rencontraient, en relation avec les hommes - cochers et postillons au premier chef - qui les exploitaient quotidiennement.

3 La notion d'exploitation trouve sens tant dans son acception première, les chevaux étant ceux dont on exploitait la force de traction dans le cadre d'une activité économique, que dans sa signification péjorative, plusieurs artistes livrant des représentations tout à fait saisissantes des «risques» ou de la "pénibilité» du «métier» de cheval dans le transport public au xix ${ }^{e}$ siècle ${ }^{1}$. Bien qu'anachroniques, ces termes empruntés au champ 
social actuel nous semblent offrir une certaine valeur heuristique pour caractériser les conditions d'exercice de ces chevaux de travail, qui apparaissent sur certaines images particulièrement rudes, sinon révoltantes, à notre sensibilité contemporaine. Dans la majorité des représentations recueillies cependant, ce sont d'autres aspects qui prédominent, parmi lesquels la puissance, la prestance ou la docilité des chevaux. Aussi tenterons-nous d'étudier ce corpus à la lumière de données issues de diverses recherches, afin d'analyser les principaux modes, ou régimes, de représentation des chevaux au travail dans ce secteur d'activité florissant.

4 Nous retracerons dans un premier temps quelques grandes lignes de la constitution du système de transport public à traction hippomobile dont le xix ${ }^{e}$ siècle constitua l'âge d'or. Puis, à partir des œuvres sélectionnées, nous explorerons le transport urbain, pour finir par le transport routier. Les œuvres choisies, centrées sur l'espace français, couvrent l'ensemble $\mathrm{du} \mathrm{xIx}^{\mathrm{e}}$ siècle, le prolongeant même un peu au-delà, au premier quart du $\mathrm{xx}^{\mathrm{e}}$ siècle, afin d'appréhender la mutation qui découle de l'apparition de l'automobile.

\section{L'édification d'un système de transport public à traction hippomobile}

5 La création de la poste aux chevaux jeta les premières bases d'un système de transport public en voiture attelée, à l'échelle de l'ensemble du royaume. Fondée à la fin $d u x^{e}$ siècle par Louis XI pour favoriser l'acheminement de ses messages, elle fut mise à la disposition du public en 1505 par Louis XII. Son organisation reposait sur un réseau de relais jalonnant des itinéraires prédéterminés, les routes de postes, qui permettaient aux voyageurs de changer de chevaux. Les chevaux étaient utilisés pour couvrir la distance entre deux relais, sous la conduite d'un ou plusieurs postillons, qui les ramenaient ensuite à leur relais d'origine. L'avantage de cette organisation était qu'elle occasionnait moins de fatigue pour les chevaux, les voyageurs pouvant par conséquent parcourir dans un temps donné des distances bien supérieures à celles qu'ils auraient effectuées avec les mêmes chevaux. Si la poste aux chevaux constituait un service public, les relais, chevaux inclus, étaient la propriété des maîtres de poste ${ }^{2}$.

6 Les maîtres de poste exercèrent leur charge à titre d'office jusqu'en 1692. Ils furent par la suite nommés par le roi ou l'administration et étaient titulaires d'un brevet officiel qui pouvait faire l'objet d'une transmission familiale, donnant lieu à la constitution de véritables dynasties de maitres de poste, ce qui était du reste encouragé en vertu de la stabilité que ces dispositions apportaient à l'organisation d'ensemble de la poste aux chevaux. Les maitres de poste devaient posséder un nombre de chevaux prescrit par l'administration, qu'ils étaient tenus de fournir en priorité aux transports de la poste aux lettres et au service du roi. En contrepartie, ils percevaient des gages, gratifications ou indemnités et bénéficiaient de certaines exemptions d'impôt, comme celle du paiement de la taille, ainsi que de privilèges d'exploitation, notamment celui d'utiliser le galop. Ils possédaient aussi le monopole, protégé par l'État, du relayage dans leur commune ${ }^{3}$.

7 C'est avec l'apparition des premières voitures de voyage publiques, à la fin du $\mathrm{XvI}^{\mathrm{e}}$ siècle, qu'un véritable service de transport public se mit en place, lorsqu'Henri III autorisa, par lettres patentes du 10 octobre 1575, l'établissement de coches par terre et par eau pour le transport des voyageurs et des bagages de Paris à Troyes, Rouen, Orléans et Beauvais. Sous Henri IV, un échelon étatique de contrôle des services de messagerie fut mis en place 
${ }^{4}$. Les messageries, qui se définissent comme des entreprises privées ou publiques de transport en commun, reliant une ville à l'autre, pouvaient soit faire usage de la poste aux chevaux, soit disposer de leurs propres cavaleries ou encore utiliser les chevaux de relayeurs libres, particulièrement sur les routes dépourvues de relais officiels ${ }^{5}$.

Hormis certaines exceptions qui s'appliquaient au XVIII ${ }^{e}$ siècle aux diligences desservant les routes de Lyon et de Lille, autorisées à utiliser le galop, un privilège qui fut plus tard étendu à l'ensemble des diligences mises en service dans le cadre de la réforme des transports de Turgot, l'allure la plus élevée admise pour les voitures des messageries était le trot enlevé. C'est le trot qui était prescrit par la réglementation au $\mathrm{XIX}^{\mathrm{e}}$ siècle, bien que les témoignages montrent que les diligences empruntaient en réalité également le pas ou le galop ${ }^{6}$.

9 Sous l'Ancien Régime, les droits de messagerie relevaient à l'origine du seul privilège du roi, qui pouvait à sa convenance les attribuer à des particuliers ou des institutions. À partir de 1676, ces droits furent rachetés par la ferme générale des Postes, nouvellement créée, qui donnait à bail les messageries à des particuliers pour une durée de six ou neuf ans. Le système de l'affermage resta en vigueur jusqu'à la Révolution, à l'exception d'une brève expérience de régie directe par l'État initiée par Turgot en 1775, dans le cadre de sa réforme des transports. Les gouvernements révolutionnaires oscillèrent entre gestion étatique et liberté d'exploitation, adoptant successivement différentes solutions à l'organisation du transport public des voyageurs. Si l'existence de la poste aux chevaux fut un temps remise en question, elle se maintint néanmoins, malgré les pénuries de chevaux et de fourrage. À partir de 1794, les messageries furent progressivement libéralisées, notamment par la loi du 9 vendémiaire an VI (30 septembre 1797), par laquelle l'État supprima la régie des messageries nationales qui avait été créée sous la Convention. Cette loi confirma également les maîtres de poste dans leur droit exclusif de relayage et fixa le montant de leurs gages ${ }^{7}$.

10 En ce qui concerne le transport public urbain, nous nous limiterons ici au cas de Paris et de sa banlieue, seule zone géographique que la collection du musée permettait d'aborder de façon satisfaisante. Dans la capitale même, deux catégories de voitures publiques virent le jour au XVII ${ }^{\mathrm{e}}$ siècle, préfigurant celles du XIX ${ }^{\mathrm{e}}$ siècle. Les premières apparurent quelque temps avant 1630, lorsqu'un certain Nicolas Sauvage, entreprenant maitre carrossier originaire d'Amiens, créa rue Saint-Martin, à l'enseigne de l'hôtel Saint-Fiacre, un service de voitures de louage. Celui-ci venait concurrencer les chaises à porteurs publiques, qui jouissaient d'une vogue considérable depuis leur mise en place en 1617. Bientôt connues sous le nom de fiacres, les voitures proposées par Nicolas Sauvage étaient de deux types, celles qui étaient hélées depuis la voie publique et celles qui étaient louées depuis la remise de leur propriétaire. À partir de la fin du XVII siècle, l'usage du terme de fiacre fut restreint au seul premier type. Ces deux types de voitures de louage devaient se maintenir jusqu'au début du $\mathrm{xx}^{\mathrm{e}}$ siècle $^{8}$.

11 Les «carrosses à cinq sols " constituent la seconde catégorie de voitures publiques parisiennes née au XVII ${ }^{\mathrm{e}}$ siècle. Tirées par deux chevaux, ces voitures à huit places circulaient, à la différence des voitures de louage, sur des itinéraires fixes et selon des horaires réguliers. Initiée par Blaise Pascal en 1662, cette première expérience de transport en commun urbain présentait plusieurs traits la rapprochant de l'organisation adoptée pour les omnibus au XIX ${ }^{\mathrm{e}}$ siècle. Les carrosses à cinq sols ne connurent cependant qu'une brève existence et un succès tout relatif, puisque leur service aurait périclité dès la première année. Ils auraient définitivement disparu en 1677, l'année 1667 étant 
également rapportée pour être celle de la cessation d'activité de l'entreprise mettant en service ces voitures. Trois raisons principales ont été avancées pour expliquer leur échec : la première est une restriction imposée par le Parlement de Paris, interdisant leur accès aux gens de petite condition, la seconde est l'augmentation des tarifs, qui passèrent rapidement de cinq sols à six, la dernière est la mort de Pascal en 1662, privant ce système de transport de sa direction'.

$\mathrm{Au} \mathrm{XVII}^{\mathrm{e}}$ et au $\mathrm{XVIII}^{\mathrm{e}}$ siècles, les liaisons entre Paris et les villes voisines étaient assurées par divers véhicules de transport en commun. Souvent de grande taille, ils pouvaient circuler sur terre, à l'instar du « carabas » ou « cabas » à la caisse d'osier tressé, ou sur les voies navigables. Utilisant la force motrice de l'eau en association avec la traction hippomobile, les coches d'eau perdurèrent jusqu'à ce que les bateaux à vapeur les remplacent à partir des années 1820-1830. La collection du musée n'en témoignant que pour le XVIII siècle, nous n'en traiterons pas ici. Bien mieux représentés y sont les coucous, qui étaient des voitures d'un plus petit modèle, apparues sous le Premier Empire, avec lesquelles on se rendait aux environs de Paris ${ }^{10}$.

13 Le développement de l'usage des voitures entraîna une diversification des types de chevaux: la notion de cheval de trait, distincte de celle de cheval porteur ou de selle, apparut à la fin du XVI ${ }^{\mathrm{e}}$ siècle et se généralisa dans la seconde moitié du XVII ${ }^{\mathrm{e}}$ siècle. Au XVIII ${ }^{\mathrm{e}}$ siècle se mirent en place des types différenciés de chevaux de trait, qui se divisaient notamment entre les chevaux de trait légers, destinés à la traction de voitures légères telles que les chaises de poste, et les chevaux de trait plus lourds, employés pour les travaux agricoles ou pour le transport de charges importantes ${ }^{11}$.

\section{Apogée et crépuscule du « cheval-moteur »}

14 C'est au XIX ${ }^{e}$ siècle que le transport public hippomobile atteignit son plein essor, tant en ville que sur les routes. André Leroi-Gourhan en souligne la contradiction apparente dans sa préface à l'ouvrage fondateur de Bernadette Lizet :

Paradoxalement, c'est le développement industriel au XIX ${ }^{\mathrm{e}}$ siècle et dans le premier tiers du $\mathrm{xx}^{\mathrm{e}}$ siècle qui marque l'apogée du «cheval-moteur ». La poste entretient alors 20000 chevaux et les omnibus de Paris, 15 000. [...] La force motrice de la vapeur est puissante mais statique et, pendant des années, le cheval de trait assurera encore la force mobile, en attendant que le moteur à essence l'élimine progressivement ${ }^{12}$.

15 Toutefois, les pics de développement du transport public routier et urbain à traction hippomobile ne furent pas concomitants, le chiffre de 20000 chevaux de poste correspondant à celui de l'année 1838 et celui de 15000 à l'effectif de l'année 1880 de la cavalerie de la Compagnie générale des omnibus (CGO). En effet, si les transports publics hippomobiles se développèrent très fortement dans les grandes villes au cours de la deuxième moitié du XIX ${ }^{e}$ siècle, la poste aux chevaux déclina dès la fin des années 1830 , sous l'effet de la construction des chemins de fer. L'utilisation des chevaux subsista néanmoins jusqu'au début $\mathrm{du} \mathrm{xx}^{\mathrm{e}}$ siècle, en complément du chemin de fer, dans le transport public inter-urbain ${ }^{13}$.

Bien que les maîtres de poste aient perdu avec la Révolution certains de leurs privilèges d'Ancien Régime et que la libéralisation des messageries les ait fragilisés, ils conservèrent un monopole de fait en matière de relayage grâce à la loi de 1805 obligeant les entrepreneurs de messagerie qui n'utilisaient pas les chevaux de poste à leur verser une 
indemnité de 25 centimes par cheval. En définitive, leur situation sociale s'éleva au cours de la première moitié du XIX ${ }^{e}$ siècle, notamment grâce à l'augmentation du service de la poste aux lettres. Agents commissionnés d'un service public, ils avaient aussi le droit de louer leurs montures à des particuliers et de créer des compagnies de transport de voyageurs. En 1834, on comptait en France 1548 relais pour 19850 chevaux, soit une moyenne de 12 chevaux par relais. Les relais étaient distants de 12 à $20 \mathrm{~km}$ et l'on relayait tous les $30 \mathrm{~km}$ environ ${ }^{14}$.

17 Deux grandes entreprises de messagerie dominaient leur secteur durant ce premier $\mathrm{XIX}^{\mathrm{e}}$ siècle : les Messageries royales et les Messageries Laffitte et Caillard ${ }^{15}$. Fondée en 1798 sous le nom d'« Entreprise générale des messageries » par des fermiers d'Ancien Régime sous la direction de la famille Nanteuil, la première reprit le mobilier et les bâtiments de l'ex-régie d'État. Après des débuts difficiles, elle connut sous le Premier Empire un essor déterminant, jusqu'à exercer un monopole sur les grands parcours. Elle conclut en 1822 un traité avec les maîtres de poste, qui reçurent le droit exclusif de fournir les chevaux des diligences de cette compagnie, en contrepartie duquel il leur était interdit de participer à une autre entreprise de messagerie. Si les Messageries royales se prévenaient ainsi contre une concurrence éventuelle des maîtres de poste, leur monopole devait néananmoins leur être disputé par l'Entreprise des messageries générales de France, Laffitte et Caillard et $\mathrm{cie}^{16}$.

18 Cette dernière naquit en 1826 de la réunion de plusieurs messageries. À sa tête se trouvaient Vincent Caillard, un employé des Ponts et Chaussées établi en 1815 comme entrepreneur de messagerie à Paris, et l'agent de change Jean-Baptiste Laffitte, un parent du banquier Jacques Laffitte, qui était également associé à la nouvelle entreprise. Elle regroupait treize routes lorsqu'en 1827, elle racheta, contre indemnités, cinq routes aux Messageries royales. Dès lors, ces deux grandes messageries s'entendirent afin de conserver leur hégémonie face aux entreprises moyennes qui existaient dans chaque région, auxquelles s'ajoutaient un très grand nombre de petites entreprises. Entre 1827 et 1839, le nombre d'entreprises de messagerie doubla, passant de 2132 à 4 652, témoignant d'une très forte croissance du trafic, liée à une bonne conjoncture économique ${ }^{17}$.

19 Cet accroissement du trafic fut aussi permis par la rénovation et l'extension du réseau routier français ainsi que par les progrès effectués en matière de construction des voitures. Le règlement du 27 septembre 1827, qui remplaçait une ordonnance de 1820, définit les caractéristiques du dernier modèle de diligence qui circula en France. À trois compartiments, ces diligences devaient être pour la première fois pourvues d'un système de frein, dit " machine à enrayer ", destiné à être actionné par le " conducteur », qui était le représentant de la compagnie à bord de la voiture. L'introduction de cette nouveauté technique fut à l'origine d'une transformation du mode de conduite des diligences, le postillon étant progressivement remplacé par un cocher. Il existait plusieurs catégories, ou classes, de diligences suivant leur nombre de places, les plus lourdes - dont le poids à vide ne devait pas excéder $2500 \mathrm{~kg}$, et en charge, 5 tonnes - étant attelées de cinq chevaux. Leur vitesse moyenne, arrêts compris, tournait autour de $6 \mathrm{~km} / \mathrm{h}$. Elle augmenta sensiblement, notamment à la faveur de haltes plus courtes, les diligences des Messageries royales atteignant $9 \mathrm{~km} / \mathrm{h}$ en $1842^{18}$.

Les voyageurs pressés et suffisamment aisés pouvaient également emprunter les mallesposte. Mises en service par l'administration postale dès 1793, ces voitures servaient avant tout à l'acheminement des dépêches et du courrier et secondairement au transport des personnes. Le terme de malle-poste ne se généralisa cependant dans la langue de 
l'administration postale que dans les années 1820. Jouissant du privilège du galop, les malles-poste constituaient le moyen de transport public le plus coûteux et le plus rapide avant le développement du chemin de fer. Leur construction se perfectionna durant la première moitié $\mathrm{du} \mathrm{XIX}^{\mathrm{e}}$ siècle et leur vitesse moyenne s'éleva nettement, passant, temps d'arrêt compris, de $4 \mathrm{~km} / \mathrm{h}$ à la fin du XvIII ${ }^{\mathrm{e}}$ siècle à $15 \mathrm{~km} / \mathrm{h}$ en $1848^{19}$.

21 Le développement du réseau ferroviaire bouleversa profondément l'organisation du système de transport public, entrainant un déclin soudain de la poste aux chevaux. En effet, une première commission parlementaire fut réunie dès 1838 afin d'examiner la question de la fermeture des relais, sans toutefois modifier leur situation. Par la suite, un crédit spécial fut voté en 1843 afin d'indemniser les relais ruinés, suivi en 1852 par une loi reconnaissant le droit à l'indemnisation des relais situés sur les axes desservis par les voies de chemin de fer, mais sans rétroactivité. La poste aux chevaux fut définitivement supprimée par une instruction du 31 mars $1873^{20}$.

Les messageries déclinèrent tout aussi rapidement à la même période. Si les Messageries royales commencèrent par chercher à s'associer au chemin de fer par un système original consistant à embarquer les diligences sur la ligne Paris-Orléans inaugurée en 1843, cette solution ne dura pas. Face à la concurrence des chemins de fer, qui captaient inexorablement les flux de voyageurs sur les axes qu'ils desservaient, laissant au transport public hippomobile les seules routes secondaires, les deux grandes messageries durent se tourner vers d'autres activités, comme les services urbains ou encore le roulage accéléré, auquel les Messageries royales se reconvertirent en 1847. En 1854, elles ne possédaient plus que 203 chevaux et 120 montures en demi-propriété lorsqu'elles rachetèrent les Messageries Laffitte et Caillard. L'entreprise abandonna définitivement le transport routier en 1875. Si l'administration des Postes se tourna vers le chemin de fer dès 1845 , avec la création d'un premier bureau ambulant pour le transport et le tri des dépêches sur la ligne Paris-Rouen, c'est surtout entre 1850 et 1855 que s'opéra le déclin des malles-poste. En 1856, on ne comptait plus que sept malles en service. Les petites entreprises qui desservaient les routes secondaires résistèrent mieux, les "grosses diligences " cédant dès lors le pas à des voitures de taille plus modeste, comme les pataches et les omnibus ${ }^{21}$.

Mais ce sont d'autres types d'omnibus qui marquèrent la renaissance du transport en commun à Paris, devenant un symbole d'égalité aux yeux de leurs contemporains et contribuant à la massification des déplacements urbains. Les différentes lignes furent exploitées concuremment par plusieurs entreprises - une dizaine en 1828, année de leur mise en service -, jusqu'à la formation en 1855 de la Compagnie générale des omnibus (CGO). Créée par décret impérial, la CGO absorba en les fusionnant l'ensemble des compagnies d'omnibus. Cette entreprise monopolistique se caractérisait par des structures de gestion modernes, qui se développèrent sous le Second Empire au sein de la CGO comme de la Compagnie impériale des voitures (CIV). Entre avril et septembre 1828, le nombre de voyages effectués en omnibus s'élevait à plus de 2,5 millions. En 1867, année où Paris accueillit une Exposition universelle, cette fréquentation atteignit 122 millions ${ }^{22}$.

Fondée en 1855 par les frères Caillard en association avec divers partenaires, la CIV se vit confier un quasi-monopole d'exploitation des voitures de louage parisiennes, notamment dans la perspective de doter la capitale d'un système de transport à même d'accueillir les millions de visiteurs attendus la même année pour l'Exposition universelle. Cependant, si la CIV se présentait presque comme une entreprise de service public, à la différence des omnibus, qui s'adressaient aux masses, le service qu'elle rendait répondait à des besoins 
plus individualisés. C'est pourquoi il fut décidé de mettre un terme à son quasi-monopole par le décret du 23 mars 1866 qui accordait à tout loueur de voiture la faculté de stationner sur la voie publique. La même année, le gouvernement lui octroya, en partie à titre de compensation, le statut de société anonyme, l'entreprise prenant dès lors le nom de Compagnie générale des voitures (CGV). Elle comptait, en 1867, 5000 voitures pour 10000 chevaux et employait près de 5000 personnes, représentant une force financière égale ou supérieure à celle de la plupart des producteurs de charbon de l'époque. À côté de ce géant, plusieurs entreprises virent le jour, dont la plus importante était la compagnie de fiacres L'Urbaine. Elle commença son activité autour de 1880 avec 700 numéros, puis connut une forte croissance, portant en moins de dix ans sa flotte à 2675 numéros et sa cavalerie à plus de 5000 chevaux $^{23}$.

Ces transformations des moyens de transport eurent aussi des incidences sur le développement des races de chevaux au XIX ${ }^{e}$ siècle. Ainsi, sous l'effet de la disparition des diligences et des nouveaux besoins nés du développement des chemins de fer, on abandonna progressivement pour les percherons la distinction entre trait léger, dont relevaient les chevaux dits "postiers", capables de soutenir un trot régulier, et trait lourd, un terme qui s'appliquait aux chevaux utilisés au pas dans le roulage ou pour le transport de matières pondéreuses. Vers 1880 , la race fut uniformisée, le nouveau percheron, d'apparence plus lourde, pouvant être employé tant pour la traction des voitures de livraison que pour celle des omnibus ${ }^{24}$.

\section{Le transport public à Paris et dans sa banlieue}

\section{Formes et allure(s) des voitures publiques urbaines}

Sous l'inscription "Voitures dites parisiennes », une affiche lithographique de la Société des voitures parisiennes (inv. CMV.1114) symbolise en quelque sorte deux des principaux types de voitures publiques rencontrées à Paris intra-muros. La première est une voiture à deux roues tenant de la chaise de poste, à caisse fermée, attelée à un cheval mené par un cocher. La deuxième est un omnibus de forme "primitive ", attelé à quatre chevaux menés par un cocher et un postillon qui est monté sur le cheval de volée de gauche. Sur cette image publicitaire, les chevaux, qui ont belle prestance, sont montrés en action. Le bai brun qui tracte la chaise de poste trotte allègrement, tandis que l'attelage de l'omnibus semble au pas, ce qui suggère peut-être le moment du départ, ou encore un ralentissement de la course. À moins qu'il ne s'agisse d'une volonté de souligner, à travers cette différence d'allure manifeste, le contraste entre la légèreté de la voiture à deux roues et le caractère bien plus massif de l'omnibus.

En effet, le pas était assigné aux voitures de roulage, tandis que le trot était l'allure réglementaire en ville pour toutes les voitures de transport de personnes, bien que l'on admît une plus grande lenteur pour les omnibus. Le trot est particulièrement bien rendu sur une aquarelle signée du peintre autrichien Gottfried Heinrich Wilda (1862-1911), datée de 1889 (fig. 1), qui représente une voiture de louage, identifiable au numéro peint à l'arrière de la caisse. Ce coupé carré, attelée de deux chevaux blancs, dégage une vive impression d'élégance, par tout un ensemble de détails, des ornements multicolores des harnais à la mise impeccable du cocher, en passant par la vaporeuse toilette claire que l'on entrevoit derrière les glaces de la voiture. Il s'agit très probablement, au regard de son caractère particulièrement soigné, d'une voiture de remise, qui pouvait être louée à la 
journée, au mois ou à la course et être remisée chez les particuliers. Bien plus nombreuses étaient les voitures de place, ou fiacres, qui stationnaient sur la voie publique et se louaient à la course ou à l'heure. À titre indicatif, 3000 d'entre elles devaient être quotidiennement en service, selon un chiffre fixé en 1865 par la municipalité. La même forme de caisse constituait l'un des deux types dominants de carrosserie utilisés pour ces voitures de place, avec, à la belle saison, des voitures ouvertes, de type milord ${ }^{25}$.

Figure 1

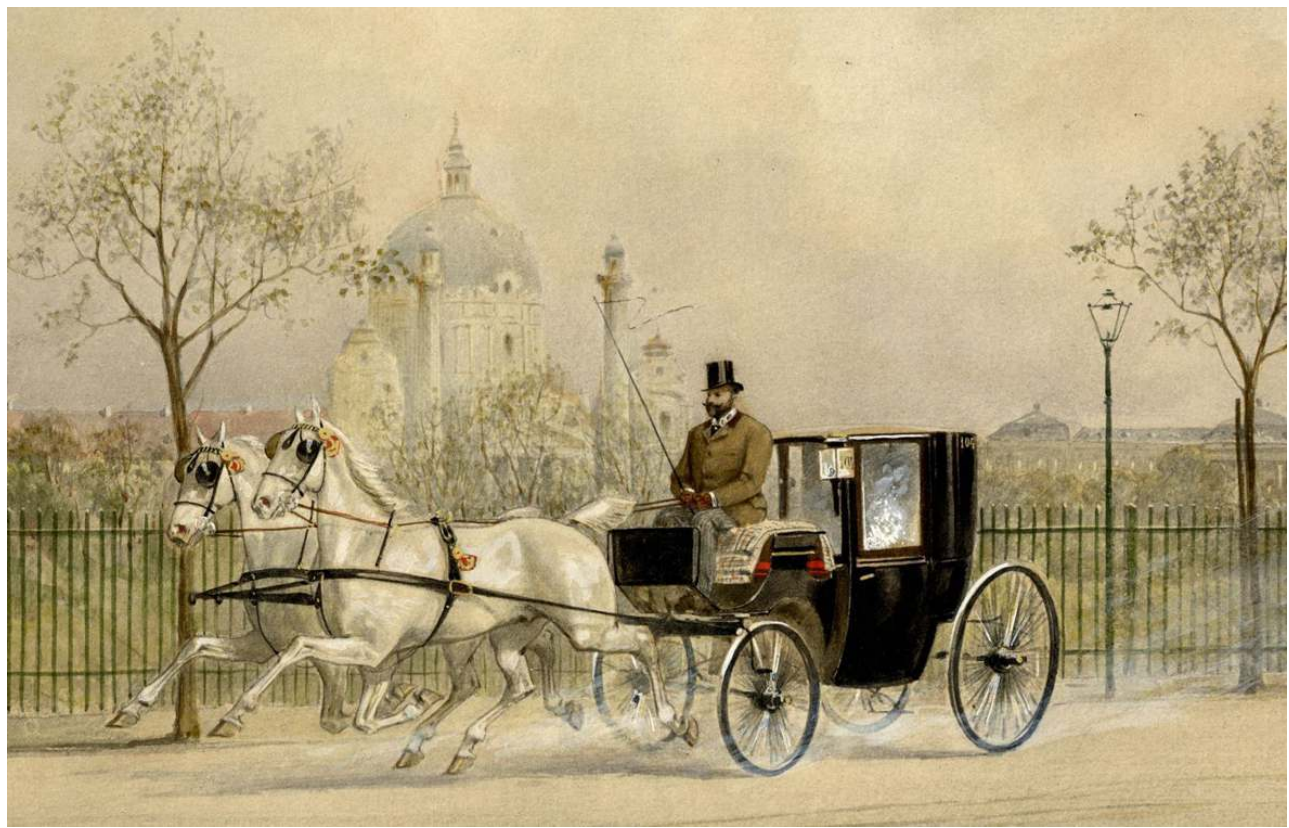

Wilda, Gottfried Heinrich (1862-1911). J.H. s. t., aquarelle, 1889, détail, inv. CMV.656.

(c) Musée national de la Voiture et du Tourisme, Palais de Compiègne.

\section{Voitures de transport en commun : coucous et omnibus}

Reliant Paris aux villes environnantes, les coucous étaient des voitures à deux roues, tractées par un cheval, pouvant transporter cinq voyageurs, dont quatre à l'intérieur et un à côté du cocher ${ }^{26}$. Cependant, ces voitures de transport en commun étaient souvent surchargées, si l'on se fie aux illustrations tirées de la collection du musée, notamment une lithographie éloquente de François Séraphin Delpech (1778-1825), d'après Charles Aubry. Intitulée Le coucou de Saint-Germain (fig. 2), elle montre sur le côté gauche une voiture si pleine que les voyageurs s'y entassent littéralement les uns sur les autres, y trouvant à peine place. Sur le côté droit, d'autres voyageurs semblent tout de même insister pour monter et n'en être dissuadés que par l'interposition d'un cocher qui pointe la voiture de son fouet, d'un geste plein d'emphase. À l'arrière-plan, le cheval attend le signal du départ, avec quelque impatience. 


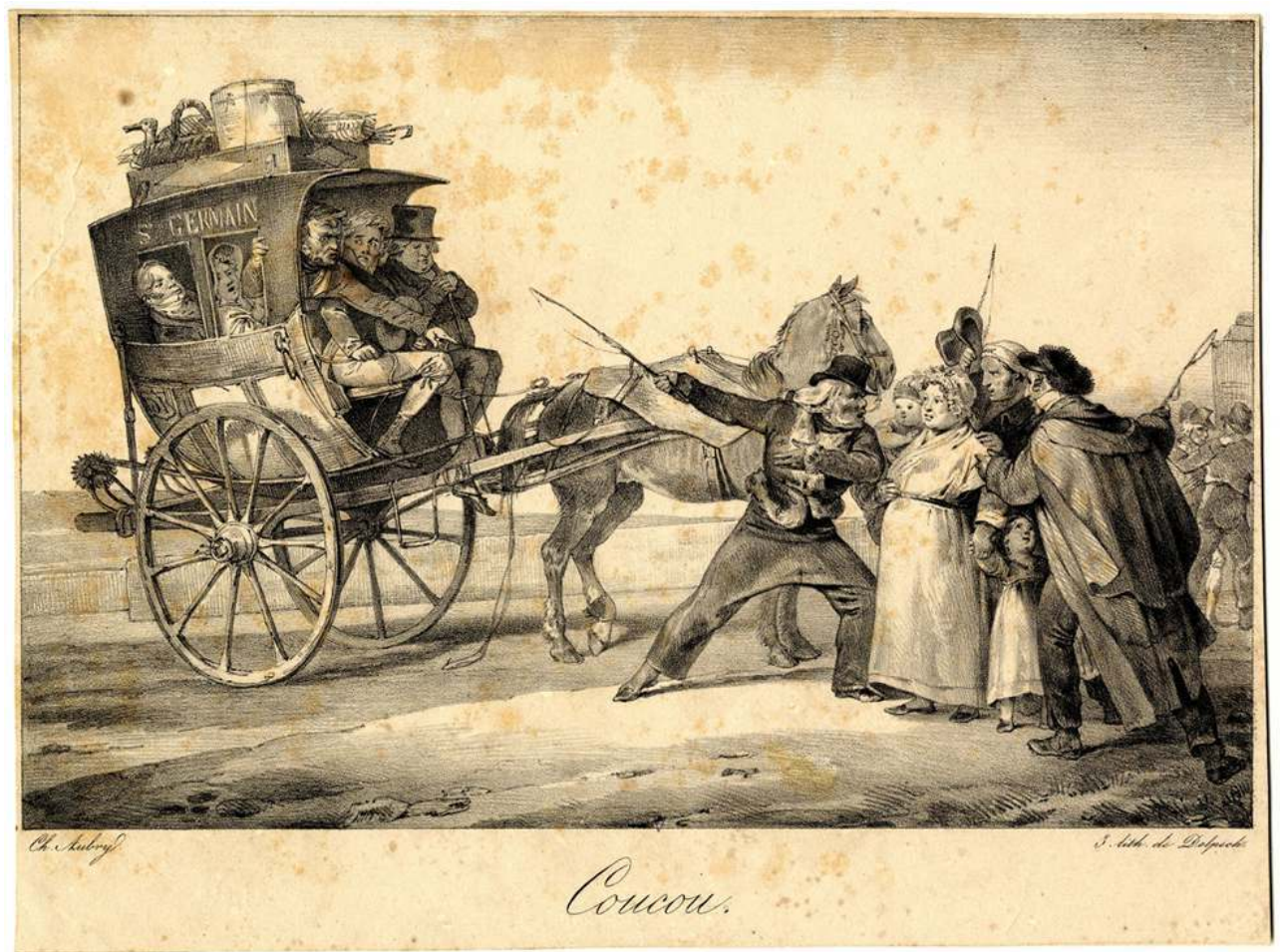

Delpech, François Séraphin (1778-1825) (lithographe), d'après Charles Aubry. Le coucou de SaintGermain, lithographie, s. d., inv. CMV.51.029.

(c) Musée national de la Voiture et du Tourisme, Palais de Compiègne.

29 À Paris, l'implantation de voitures de transport en commun demanda une certaine persévérance. De nombreuses entreprises sollicitèrent dès 1819 l'autorisation d'y faire circuler de telles voitures, sans toutefois aboutir. En 1828, le nombre de demandes rejetées s'élevait à près de 300 , refus motivés par la crainte que ces voitures ne perturbent la circulation, mais aussi, en filigrane, par la peur du «désordre démocratique » que pourraient introduire des voitures destinées à toutes les classes de la société. C'est finalement à Stanislas Baudry et à Louis Marie Debelleyme, préfet de police de sensibilité plus libérale que son prédécesseur, que revient la création des omnibus parisiens. Ancien chirurgien engagé dans l'armée napoléonienne, reconverti sous la Restauration en entrepreneur dynamique, Stanislas Baudry avait instauré un premier service de voitures publiques à Nantes en 1826 . Fort du succès de ces voitures vite qualifiées d'omnibus, il avait lui aussi requis en vain l'autorisation d'en établir à Paris puis s'était associé à Eugène Théodore Boitard et Antoine Rousselot de Saint-Céran, fondant le 29 et 30 août 1827 l'Entreprise générale des voitures dites omnibus. Après les avoir introduites à Bordeaux et Lyon, ils reçurent en janvier 1828 l'autorisation d'« établir dans l'intérieur de Paris jusqu'à concurrence de cent voitures publiques à destination fixe, dites omnibus ${ }^{27} »$.

L'arrêté préfectoral précisait notamment que les voitures devaient être «à quatre roues, traînées par deux chevaux ou par trois au plus, attelés de front ». L'Entreprise générale des omnibus aurait exploité 10 lignes dès l'année 1828, bientôt rejointe par d'autres sociétés. En 1830, dix compagnies utilisaient une quarantaine de lignes avec une centaine de véhicules au total et un trafic moyen de cent voyageurs par voiture et par jour ${ }^{28}$. Une 
remarquable série de six lithographies, exécutées d'après des dessins de Raffet et datées de 1828 et 1829 , se fait l'écho de l'événement que représenta la mise en circulation de telles voitures.

Figure 3

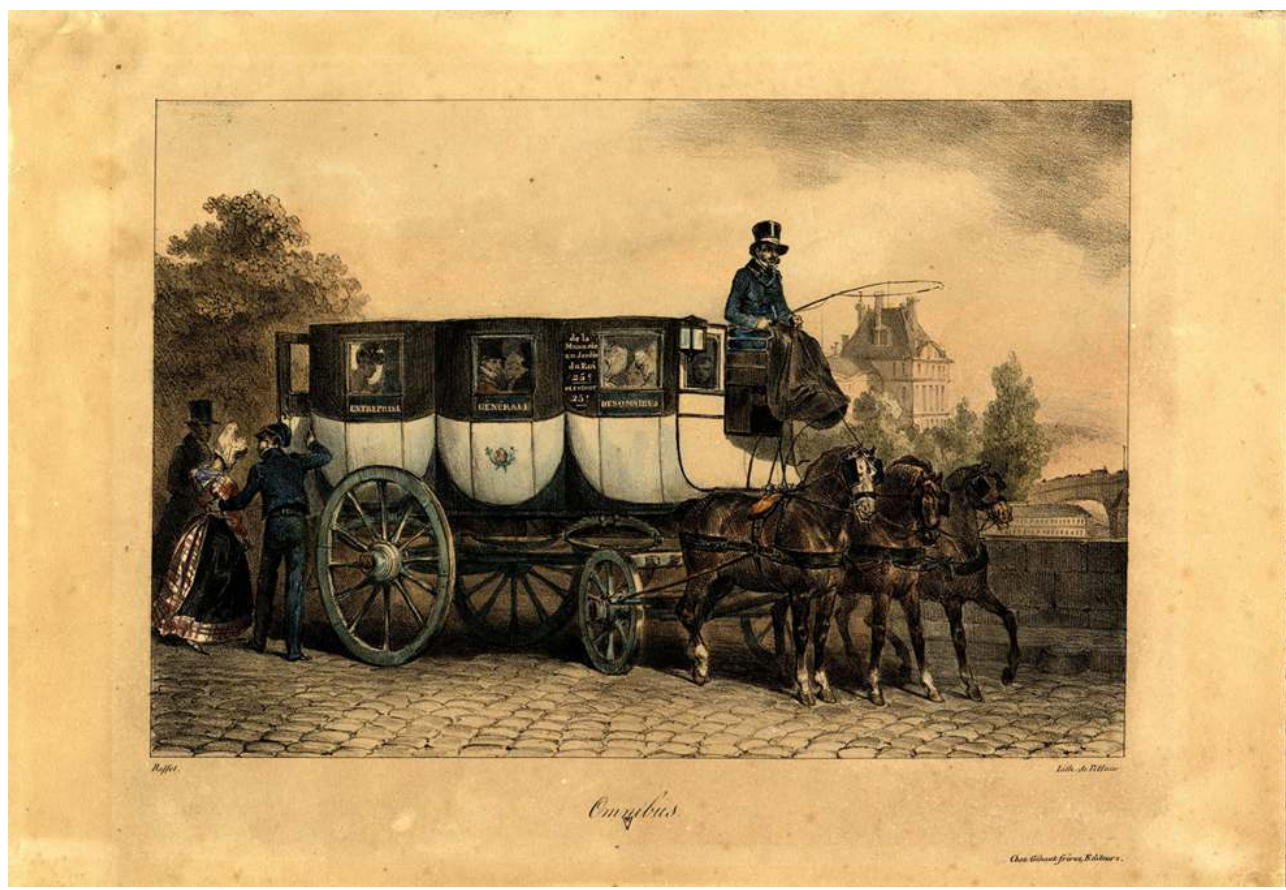

Villain (lithographe), d'après Raffet. Omnibus de l'Entreprise générale des omnibus, lithographie, 1828, inv. CMV.1123.

(c) Musée national de la Voiture et du Tourisme, Palais de Compiègne.

31 Une sélection de quatre d'entre elles permet de rendre compte des principales variations rencontrées en matière de carrosserie et d'attelage. Toutes ces voitures ont une caisse de forme identique, parallélépipédique, et dotée de trois fenêtres sur chaque côté. Cependant, elles se différencient par leur structure et leur décor. Les omnibus de l'Entreprise générale des omnibus (fig. 3) et de la société Les Écossaises sont divisés en trois compartiments, tout comme les diligences de cette époque, tandis que ceux de la compagnie Les Béarnaises et du "Service public des tricycles" ont une caisse sans division, dont la forme rectangulaire est accentuée par des registres de couleurs différentes. Les Tricycles (fig. 4) sont les seuls omnibus à trois roues, tandis que pour ce qui est des types d'attelages, toutes les voitures sont attelées en paire, à l'exception de celle de l'Entreprise générale des omnibus, dont l'attelage est à trois chevaux de front. 


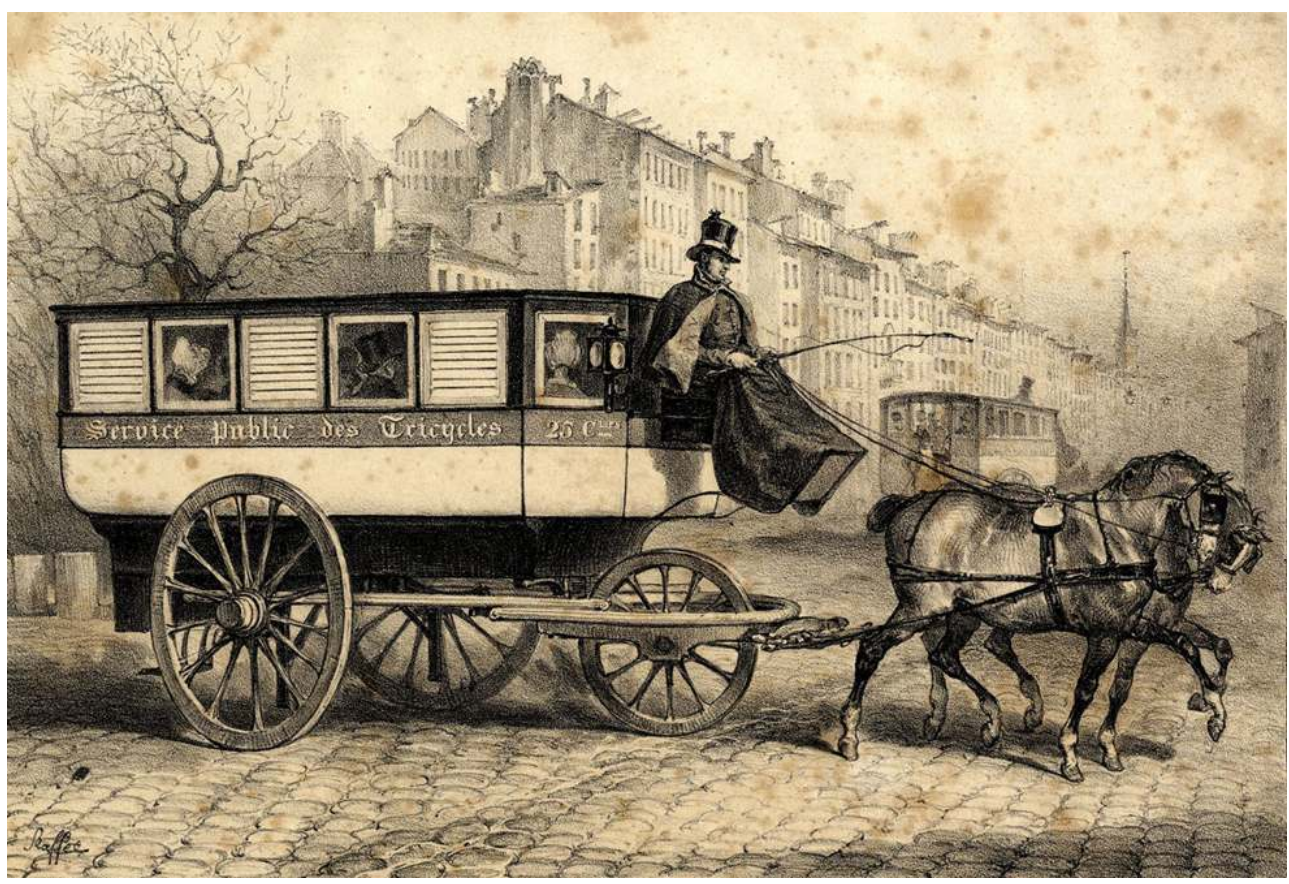

Villain (lithographe), d'après Raffet. Tricycle, lithographie, 1828, détail, inv. CMV.1122.

(c) Musée national de la Voiture et du Tourisme, Palais de Compiègne.

Sur chacune de ces lithographies, les chevaux paraissent tous assez fortement contraints, la tête très relevée et le front en avant, et semblent avancer à une allure lente, au pas plutôt qu'au trot. Ces attitudes physiques pourraient être voulues par le dessinateur luimême, qui donnerait ainsi une représentation stéréotypée des chevaux en action, tout comme elles pourraient traduire la pénibilité du travail des chevaux d'omnibus, soumis à un effort de traction particulièrement important et amenés à s'arrêter de façon très fréquente, à la demande de leurs passagers (les arrêts fixes n'apparurent qu'en 1899). La vitesse moyenne des omnibus, limitée dans les premiers temps à $7 \mathrm{~km} / \mathrm{h}$, s'élevait à $8 \mathrm{~km} /$ h, arrêts inclus, pour la période $1855-1869^{29}$.

Tirée de la série "La voiture à Paris", une photographie d'Eugène Atget (1857-1927) montre un omnibus de la ligne La Villette-Saint Sulpice à l'arrêt, vide de toute présence humaine, seulement animé par son attelage de trois chevaux se tenant au repos (fig. 5). Datée de 1910, elle revêt également un intérêt documentaire notable, saisissant cette fois la fin de la période d'exploitation des omnibus, remplacés par des autobus entre 1905 et $1913^{30}$. La ligne La Villette-Saint Sulpice fut du reste la dernière à disparaitre, avec un ultime parcours effectué le 11 janvier 1913, célébré par les industriels de l'automobile. La même année marqua également la disparition du tramway hippomobile ${ }^{31}$. 


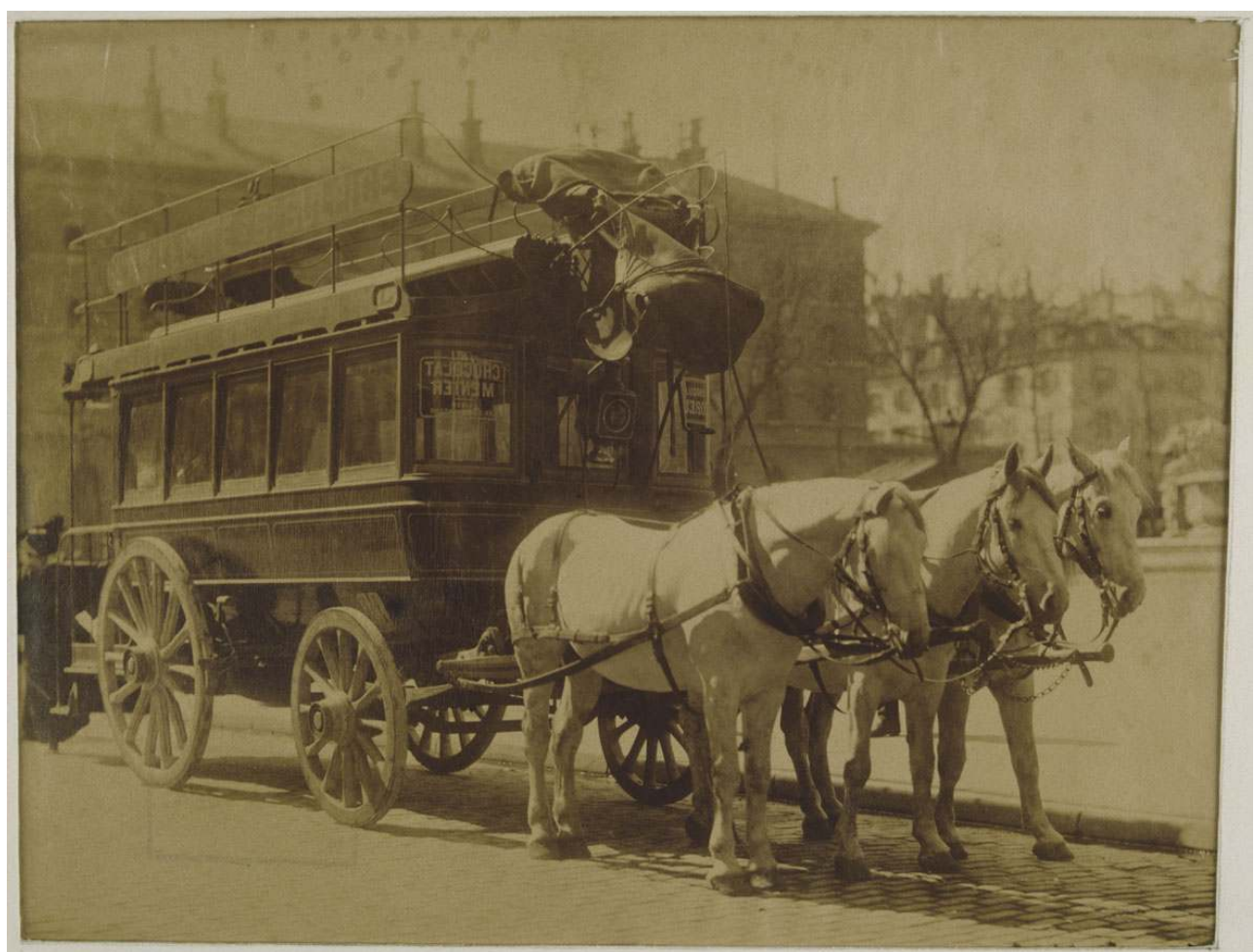

Atget, Eugène (1857-1927). Omnibus « La Villette-Saint-Sulpice », série La voiture à Paris, photographie, 1910, inv. CMV.2012.0.009

Phot. Arnaudet, Daniel, RMN-Grand Palais (domaine de Compiègne). (c) Agence photographique de la Réunion des Musées Nationaux : 94-057176. N.

La confrontation entre la photographie d'Atget et les lithographies représentant les premiers omnibus fait apparaitre des continuités en matière de carrosserie : du début à la fin, les omnibus présentent une forme de caisse globalement inchangée, surmontée cependant d'une impériale depuis 1853 . On pourrait également déduire de la similitude entre l'attelage photographié par Atget et celui de la lithographie représentant la voiture de l'Entreprise générale des omnibus que, d'abord minoritaire, l'attelage à trois chevaux de front se soit par la suite généralisé. Si ce type d'attelage paraît bien avoir été dominant dans la seconde moitié $d u$ XIX ${ }^{e}$ siècle et au début du Xxe siècle, on sait cependant que cette continuité ne fut pas linéaire, le nombre de chevaux de l'EGO ayant été pour un temps réduit à deux en 1834, tandis que la CGO mit en service à la fois des omnibus à deux et à trois chevaux, en fonction de leur nombre de places. Des rapprochements peuvent aussi être établis à propos de la robe des chevaux, qui sont, à l'exception de celle des chevaux de l'Entreprise générale des omnibus, toutes de couleur blanche ou gris clair. Ces couleurs de robe constituent une caractéristique des percherons, qui formaient la cavalerie de nombreuses entreprises de transport, et notamment de la Compagnie générale des omnibus $^{32}$.

Enfin, un détail présent sur la photographie d'Atget retient l'attention. Il s'agit du cheval du premier plan, qui est aveugle. C'est sans doute pour l'effet d'étrangeté induit par cette particularité physique qu'Atget aura choisi de photographier cet attelage, mais aussi probablement, afin de "relever [un indice] [...] pour le procès de l'histoire ", pour reprendre la terminologie de Walter Benjamin. Il se fait témoin d'une pratique qui 
permettait de tirer parti jusqu'au bout des chevaux, en dépit de leurs tares : la Compagnie générale des omnibus (CGO) attelait ainsi les chevaux aveugles avec des chevaux valides ${ }^{33}$.

\section{«Les risques du métier » de cheval dans le transport public urbain}

Si la photographie d'Atget peut se lire comme l'image d'une utilisation raisonnée de la force de travail des chevaux, plusieurs œuvres de la collection attestent de situations nettement plus dramatiques. Toutes témoignent des conditions de travail auxquelles étaient soumis les chevaux utilisés dans le transport public urbain, dont on sait qu'elles constituèrent l'un des facteurs déclencheurs d'un mouvement d'opinion en faveur d'un meilleur traitement des animaux, justifiant la naissance de la Société protectrice des animaux et le vote en 1850 de la première loi de protection des animaux domestiques, dite loi Grammont. Celle-ci fut la première à envisager la protection des animaux au nom du respect de l'animal pour lui-même et non, comme c'était le cas dans le cadre de la loi du 28 septembre 1791, au titre du préjudice subi par son propriétaire du fait de la dépréciation ou du décès de l'animal ${ }^{34}$.

En effet, les chevaux employés dans le transport public s'usaient prématurément, d'abord en raison des spécificités du travail qu'ils devaient fournir, les allures vives qui leur étaient imposées, même de courte durée, étant de nature plus éprouvante que la traction de charges plus lourdes à des allures plus lentes. Ainsi, un cheval de mine avait-il une carrière environ deux fois plus longue qu'un cheval employé à la traction des omnibus de la CGO. Cependant, le travail des chevaux de fiacre était, par comparaison avec celui des chevaux d'omnibus, sensiblement plus difficile. En effet, les chevaux d'omnibus travaillaient sur des parcours presque toujours identiques et à horaires fixes, couvrant en 1869 une distance moyenne de $16,5 \mathrm{~km}$ par jour en trois heures et demie ou quatre heures. Les chevaux de fiacre effectuaient quant à eux des parcours libres, sur des distances et à des vitesses variables, sans bénéficier de repas ou de moments de récupération réguliers. À la fin du XIX ${ }^{e}$ siècle, un fiacre de la CGV parcourait en moyenne $60 \mathrm{~km}$ en seize heures. Les conditions de travail des chevaux de fiacre dépendaient également davantage de leur cocher ${ }^{35}$. 


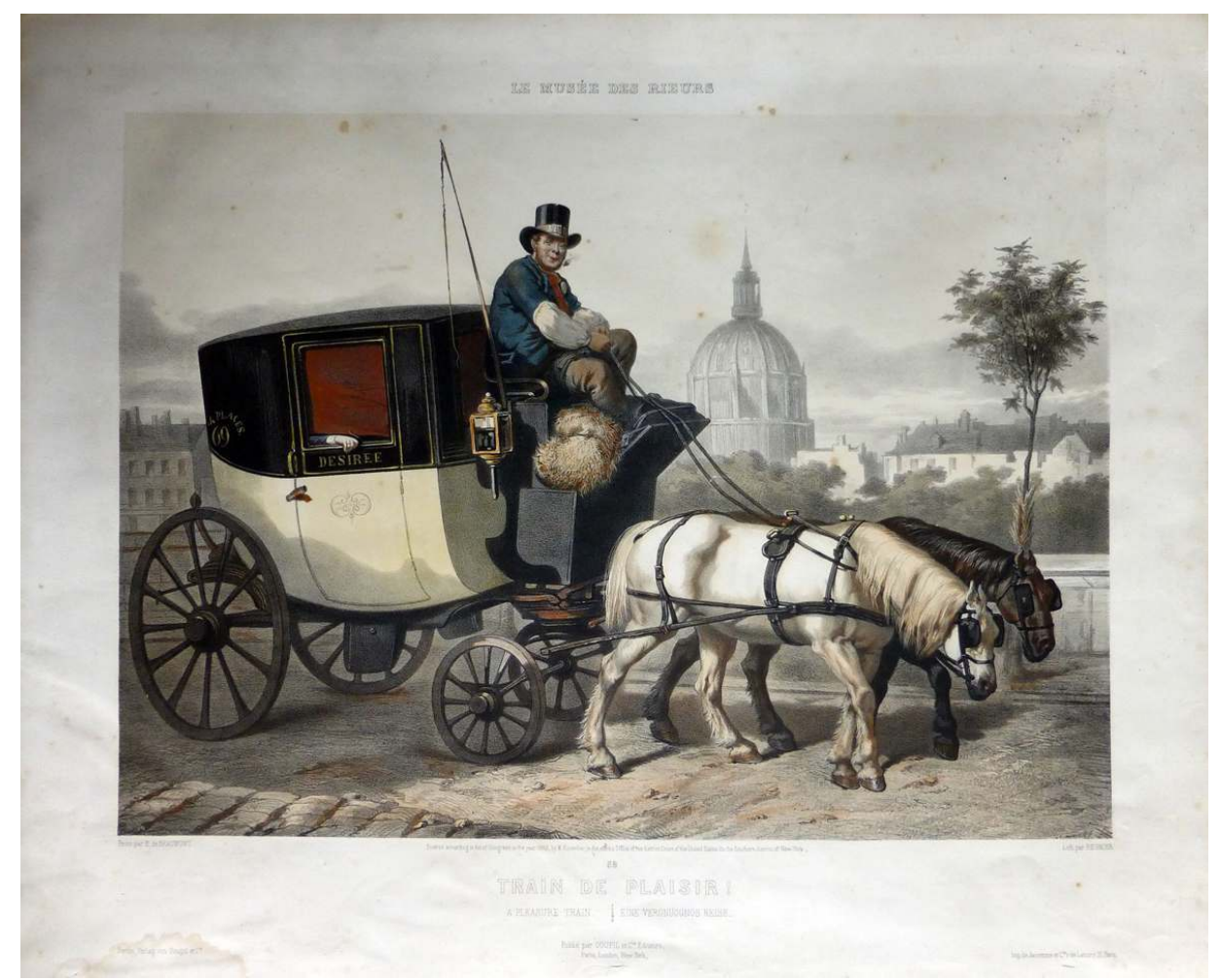

Regnier (lithographe), d'après Beaumont, Charles Édouard de (1821-1888). Fiacre, Le Musée des Rieurs, Série «Train de plaisir », Goupil édit., lithographie, dernier quart du XIXe siècle, inv. CMV.1115. (c) Musée national de la Voiture et du Tourisme, Palais de Compiègne.

L'épuisement constituait l'un des principaux risques encourus par les chevaux de fiacre. Il est montré par une lithographie à la lettre ironique, sinon grivoise, que l'on peut dater du dernier quart du XIX ${ }^{e}$ siècle (fig. 6). Encadrée par les inscriptions « Le musée des rieurs » et «Train de plaisir », l'image met en scène un coupé de forme ventrue, portant sur la portière le nom de " Désirée », tracté par une paire de chevaux à la peine, tête basse. Bien qu'ils paraissent correctement nourris, leurs signes d'épuisement tranchent avec l'attitude du cocher, qui tourne la tête vers le spectateur, un demi-sourire aux lèvres. Un risque plus grave encore, la défaillance mortelle en cours de travail, est illustré par une toile spectaculaire du peintre lyonnais Nicolas Sicard (1840-1920). Sous le titre Un accident (fig. 7) et datée de 1882, elle prend pour sujet un fiacre de la compagnie L'Urbaine, reconnaissable à sa caisse jaune et noire. L'un de ses deux chevaux s'est effondré, sans doute après avoir glissé sur le sol neigeux. Le poids du coupé, chargé de bagages, a peutêtre contribué à cette chute. Un attroupement de badauds s'est formé autour de la scène de l'accident, un homme retenant le cheval valide, tandis qu'un employé de boucherie et le cocher s'emploient à dételer le cheval défaillant, dont les nasaux sont encore fumants. Une vive émotion se dégage de cette scène, véhiculée par le contraste entre le corps massif du cheval à terre, impuissant, et le spectre des attitudes humaines autour de lui, qui va du comportement rationnel et sans états d'âme du cocher à l'intense curiosité du jeune garçon sur la gauche, en passant par le personnage féminin située sur la droite, dont la main portée au visage suggère l'émotion. 


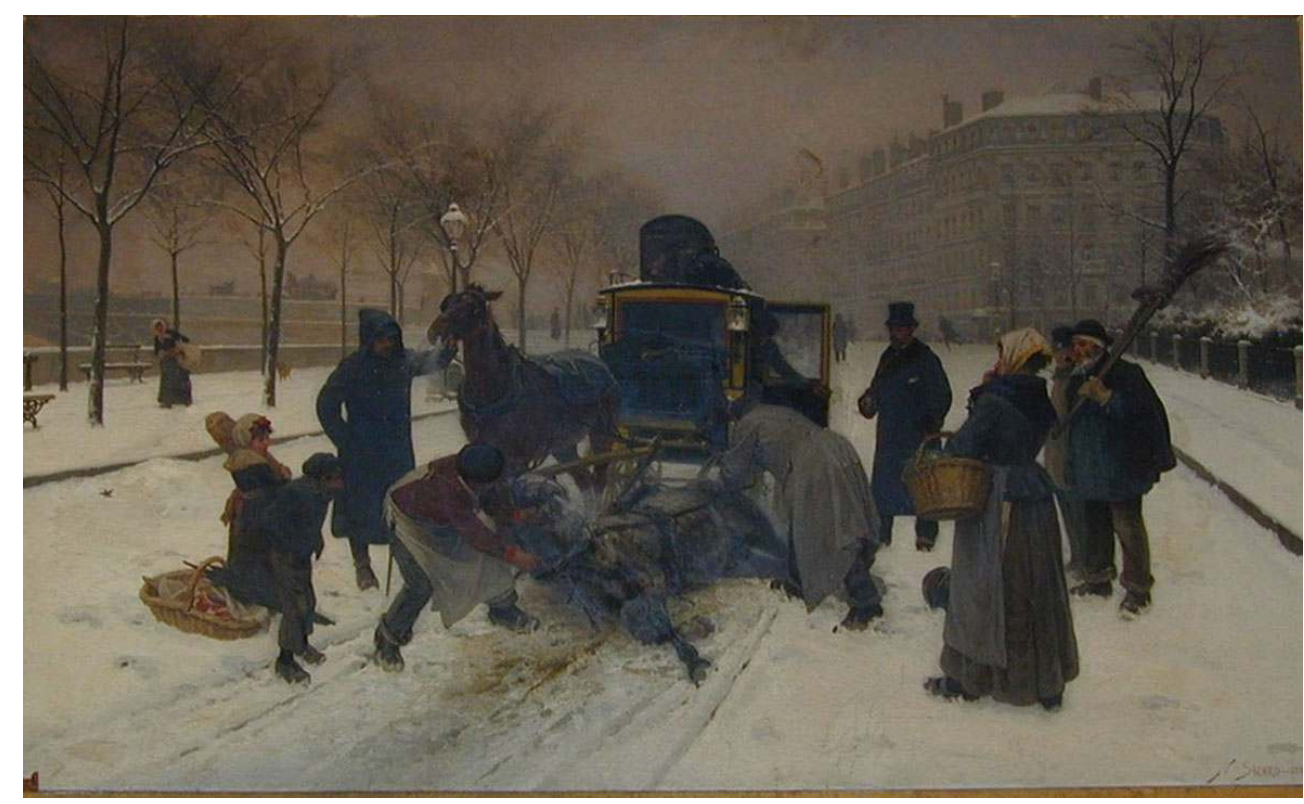

Sicard, Nicolas (1840-1920). Un accident, huile sur toile, 1882, dépôt du musée Taire, Niort, inv. CMV.58.D.1.

Phot. Poirier, Marc. (C) Musée national de la Voiture et du Tourisme, Palais de Compiègne.

Toutefois, c'est le sort des chevaux de coucous durant la première moitié du XIx siècle qui apparaît le plus déplorable, au regard de la collection du musée. La Route de Saint-Cloud, gravée par Philibert-Louis Debucourt (1755-1832) d'après un dessin d'Antoine Charles Horace Vernet, dit Carle (1758-1832) (inv. CMV.1257), montre un coucou chargé à sa pleine capacité, auquel est attelé un cheval efflanqué, représenté au moment précis où il vient de broncher. La voiture semble agitée par un cahot soudain, une expression de surprise émane de l'ensemble des protagonistes, cheval, cocher et passagers confondus, tous paraissant pris au dépourvu par ce faux-pas.

La vie d'un cheval (fig. 8), due au peintre et prolifique lithographe Victor-Jean Adam (1801-1866), affiche un certain nombre de similitudes formelles avec l'image précédente. Le coucou, à la caisse peinte des mêmes couleurs, est également représenté venant de la gauche. Il est cette fois considérablement surchargé et attelé à une haridelle plus pitoyable encore, forçant sur le poitrail, tête très basse, bouche ouverte et baveuse. Bien que son cheval soit manifestement exténué et fort probablement malade (on pense à la morve), le cocher lève son fouet pour qu'il continue d'avancer, dans l'indifférence des passagers. À l'arrière-plan à droite surgit une voiture particulière tractée par un fringant cheval, comme pour mieux souligner l'écart entre l'existence choyée d'un cheval de luxe et le travail infernal des chevaux desservant les environs de Paris. La présence d'un cheval mort, en arrière-plan à gauche, emporté par un équarisseur, paraît ainsi symboliser la fin proche du sujet principal de la scène. 


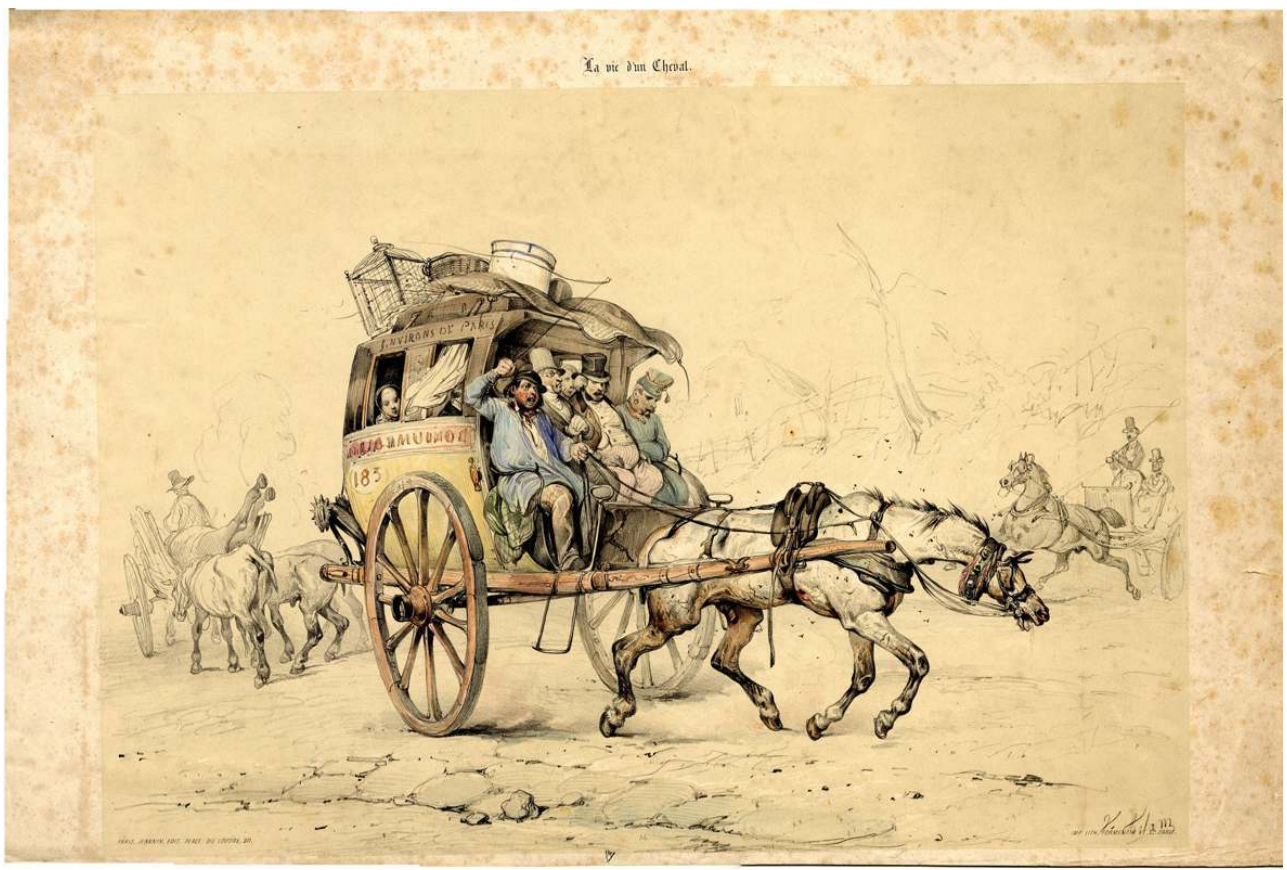

Formentin \& Cie (imprimeur-lithographe), Adam, Victor-Jean (1801-1866). La vie d'un cheval, lithographie, s. d., inv. CMV.3690.

Phot. Musée national de la Voiture et du Tourisme, Palais de Compiègne. @ Musée national de la Voiture et du Tourisme, Palais de Compiègne.

Une troisième œuvre, intitulée Les petits bourgeois parisiens en partie de campagne ou Le dîner renversé (fig. 9), se gausse de la mésaventure survenue à quelques représentants de cette classe sociale ascendante, qui accédait à une nouvelle mobilité. Cette fois, le cheval a trébuché, renversant une passagère et les provisions de bouche. Seul un chien, un gigot dans la gueule, y trouve son compte. Si la caricature fait montre d'ironie à un premier niveau à l'endroit des passagers, elle prend aussi pour cible le cocher, qui est, comme précédemment, représenté le bras levé sur son cheval, ne trouvant que le fouet pour l'amener à se relever. Cette image est certainement celle qui paraît la plus révoltante aujourd'hui, en raison de la brutalité dont le cocher fait preuve envers son cheval affaibli. Elle fait écho à la mauvaise réputation qui était attachée aux cochers et charretiers, fortement décriés pour les mauvais traitements qu'ils infligeaient à leurs chevaux ${ }^{36}$. 


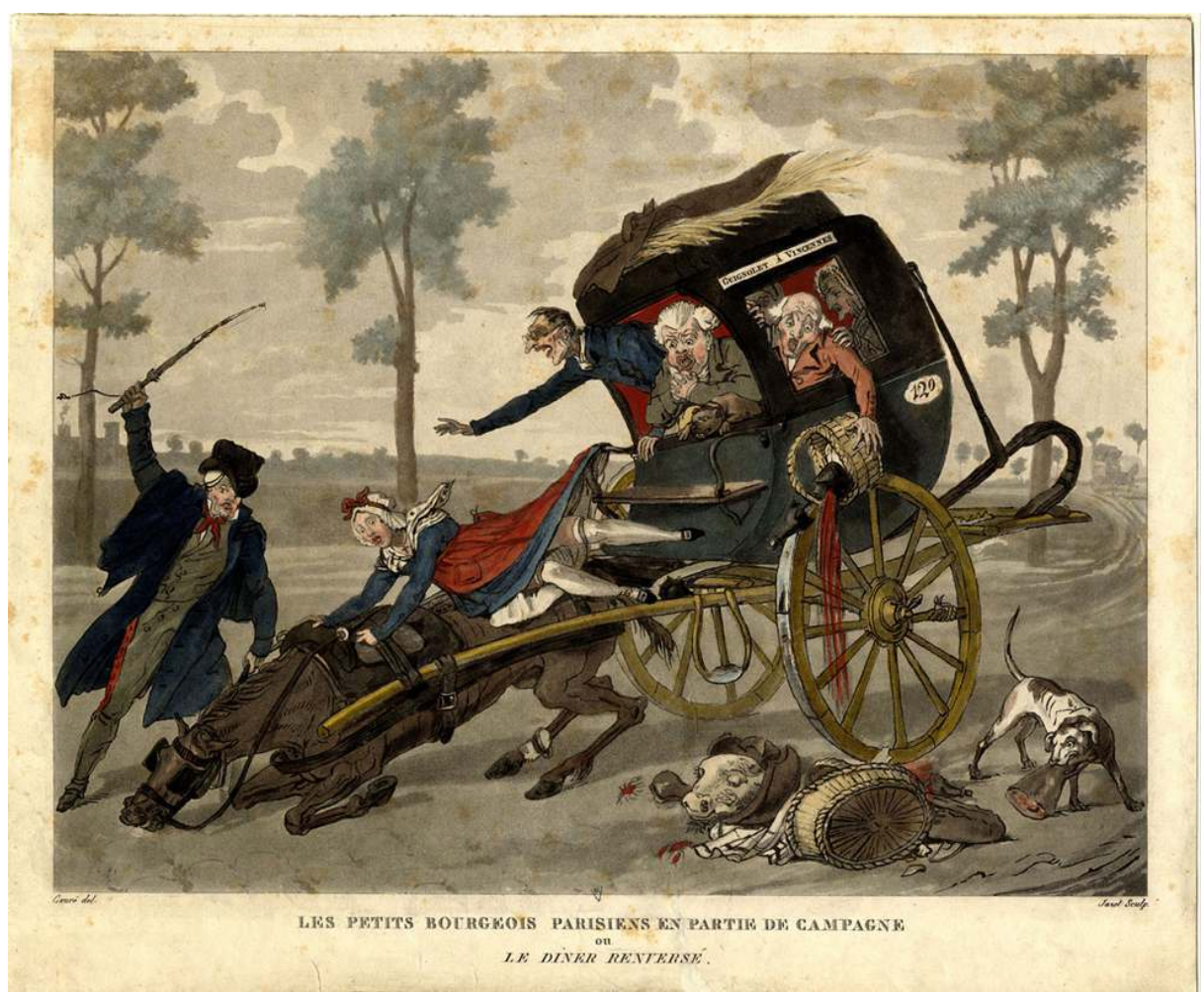

Jaxet (graveur), d'après Coeuré. Les petits bourgeois parisiens en partie de campagne ou le dîner renversé, gravure coloriée, s. d., inv. CMV.55.002.27.

Phot. Musée national de la Voiture et du Tourisme, Palais de Compiègne. (c) Musée national de la Voiture et du Tourisme, Palais de Compiègne.

Un dernier facteur déterminant les conditions de travail des chevaux résidait dans les modes de gestion des entreprises dont ils relevaient, lesquels étaient aussi à mettre en relation avec la taille de ces entreprises. Ainsi, sauf exception, on peut déduire que les entreprises de transport qui avaient une cavalerie importante traitaient leurs chevaux convenablement ${ }^{37}$.

\section{Le transport public routier}

\section{Sur les routes de poste}

La page de couverture de la partition d'une chansonnette consacrée au personnage du postillon (fig. 10), véritable cheville ouvrière de l'organisation de la poste aux chevaux, le campe juché sur le cheval porteur d'un attelage à deux chevaux, dans son uniforme caractéristique, ses chevaux fermement tenus à l'arrêt. Bien que la représentation de l'uniforme soit dans l'ensemble exacte, l'artiste s'est trompé quant à la place du brassard, qui est figuré sur le bras droit, alors que le postillon devait réglementairement le porter au bras gauche. Cette erreur pourrait être due à la raréfaction de la figure du postillon, qui appartient déjà presque au passé en 1857, année de la publication de cette chansonnette composée par Paul Henrion (1819-1901) sur des paroles de Félix Mouttet. Ainsi, par la relation instaurée entre la figure centrale du postillon et la locomotive 
représentée à l'arrière-plan, cette image nous paraît-elle symboliser le devenir de la poste aux chevaux au xIX ${ }^{e}$ siècle, dont l'apogée fut suivi d'un déclin extrêmement rapide ${ }^{38}$.

Figure 10

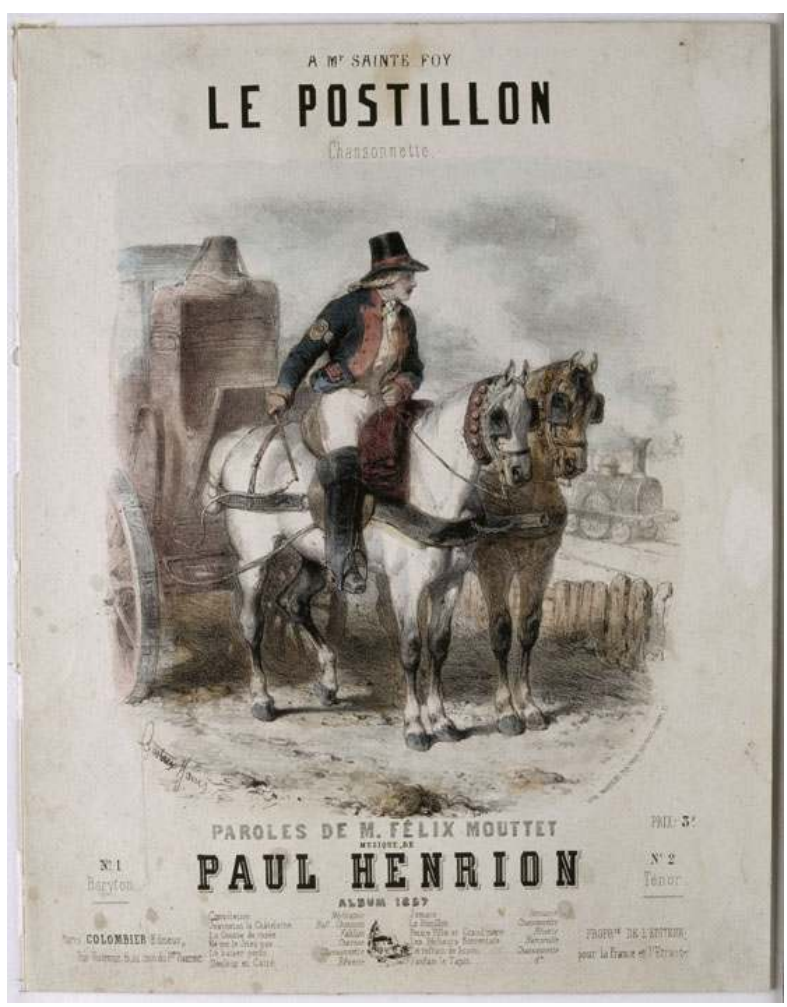

Gustave, James. Le postillon, page de couverture de partition, sur des paroles de Félix Mouttet et une musique de Paul Henrion, s. d., inv. CMV1119.

Phot. Arnaudet, Daniel, RMN-Grand Palais (domaine de Compiègne). (c) Agence photographique de la Réunion des Musées Nationaux : 96-008918.

Cet apogée se reflète dans une lithographie d'Engelman, d'après Bernard Edouard Swebach (1800-1870), intitulée Dormeuse de poste (CMV.3665), qui représente une voiture de voyage privée emblématique du premier tiers du XIX siècle, attelée à quatre chevaux et menée par deux postillons. Les nuages de poussière soulevés par l'attelage contribuent à l'effet de puissance produit par les chevaux, montrés en pleine course, dans un galop que paraît vouloir freiner le postillon monté sur le timonnier. En arrière-plan, un autre postillon chevauche sa monture pour la ramener au relais.

\section{L'âge d'or des diligences}

C'est aussi une impression de puissance et de célérité qui se dégage des représentations d'attelages de voitures publiques de la même époque. Datée 1823, la Diligence des Messageries royales de la ligne Rouen-Le Havre, lithographiée par Loeillot-Hartwig (1788-?), d'après Benard (fig. 11), représente l'une des premières formes de la diligence à trois compartiments, qui incarne l'âge d'or du transport public routier hippomobile. À l'avant se trouve une banquette abritée par une capote, puis le compartiment dit de berline ou d'intérieur, et à l'arrière, le compartiment de rotonde. Sur l'impériale se trouvent également des places pour le conducteur et trois voyageurs. La composition est structurée par la diagonale formée par la voiture et son attelage à cinq chevaux en 
arbalète, dirigé du fouet par un postillon. Les chevaux semblent littéralement débouler vers le spectateur, probablement dans un trot enlevé, voire dans un galop, bien que cette allure fût théoriquement interdite aux voitures des messageries ${ }^{39}$.

Figure 11

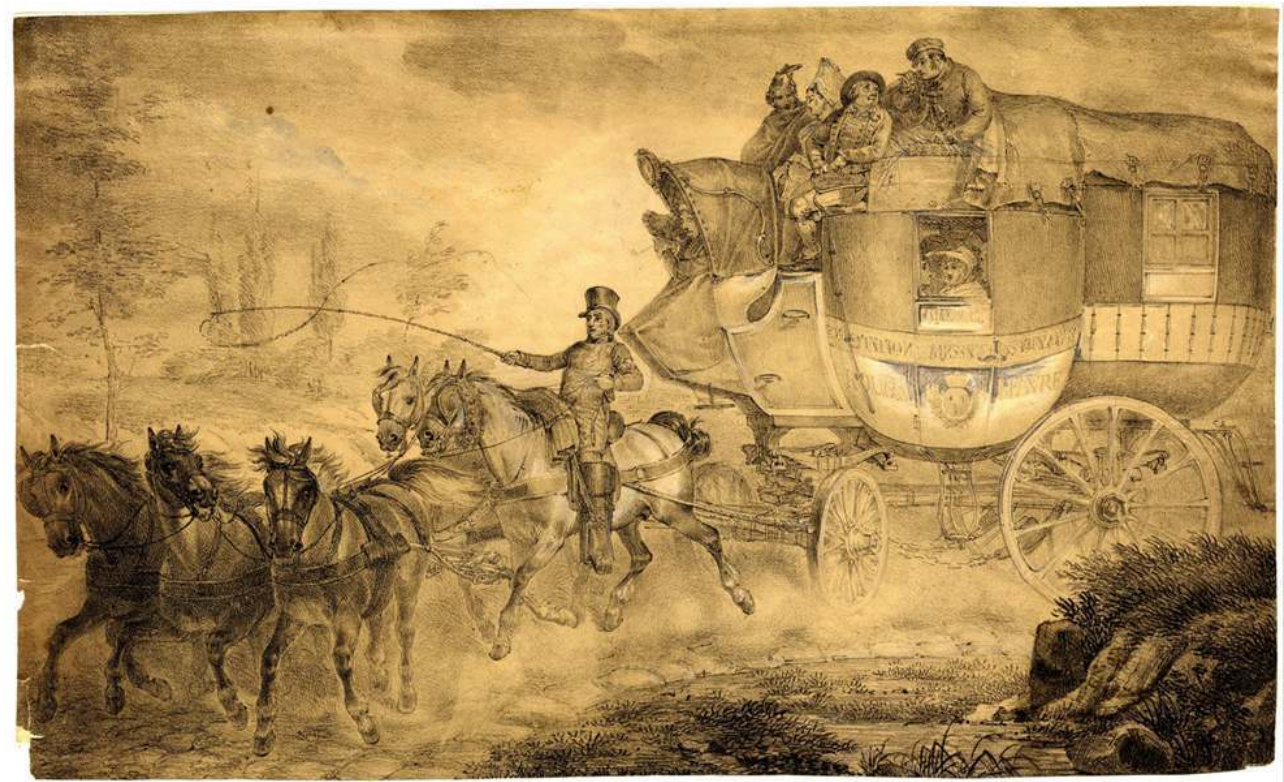

Benard, A. (?). Diligence des Messageries royales de la ligne Rouen-Le Havre, lithographie rehaussée, vers 1820, inv. CMV.3762.

Phot. Musée national de la Voiture et du Tourisme, Palais de Compiègne. (c) Musée national de la Voiture et du Tourisme, Palais de Compiègne.

Les «jumelles », exploitées par une messagerie de taille moyenne, desservaient la route Paris-Valenciennes-Bruxelles-Sedan. Une lithographie de Ch. Villain, d'après Loeillot, datée 1824, met en scène l'une de ces voitures. Il s'agit cette fois d'une forme de transition qui précède le type achevé de la diligence à trois compartiments, un véritable compartiment de coupé s'étant substitué aux places avant représentées sur l'œuvre précédente. Cette voiture est elle aussi tractée par un attelage en arbalète mené par un postillon. Il est à noter toutefois une particularité du harnachement des chevaux, qui portent ici des colliers alors que ce sont des bricoles qui figurent sur toutes les autres représentations relatives au transport routier. Cette exception pourrait s'expliquer par un choix particulier de la compagnie, ou bien une erreur de l'artiste ${ }^{40}$.

47 Une lithographie intitulée Les Messageries générales de France Laffitte, Caillard \& Cie (fig. 12), signée Victor-Jean Adam, représente le type achevé de la diligence à trois compartiments. Elle est conduite par un cocher, en grandes guides. Cette œuvre est tout à fait caractéristique du style de Victor-Jean Adam, qui allie un trait énergique à une prédilection pour les courbes dessinées par de rondes encolures, produisant un effet de contraste dynamique particulièrement approprié à la représentation des chevaux en pleine course. 


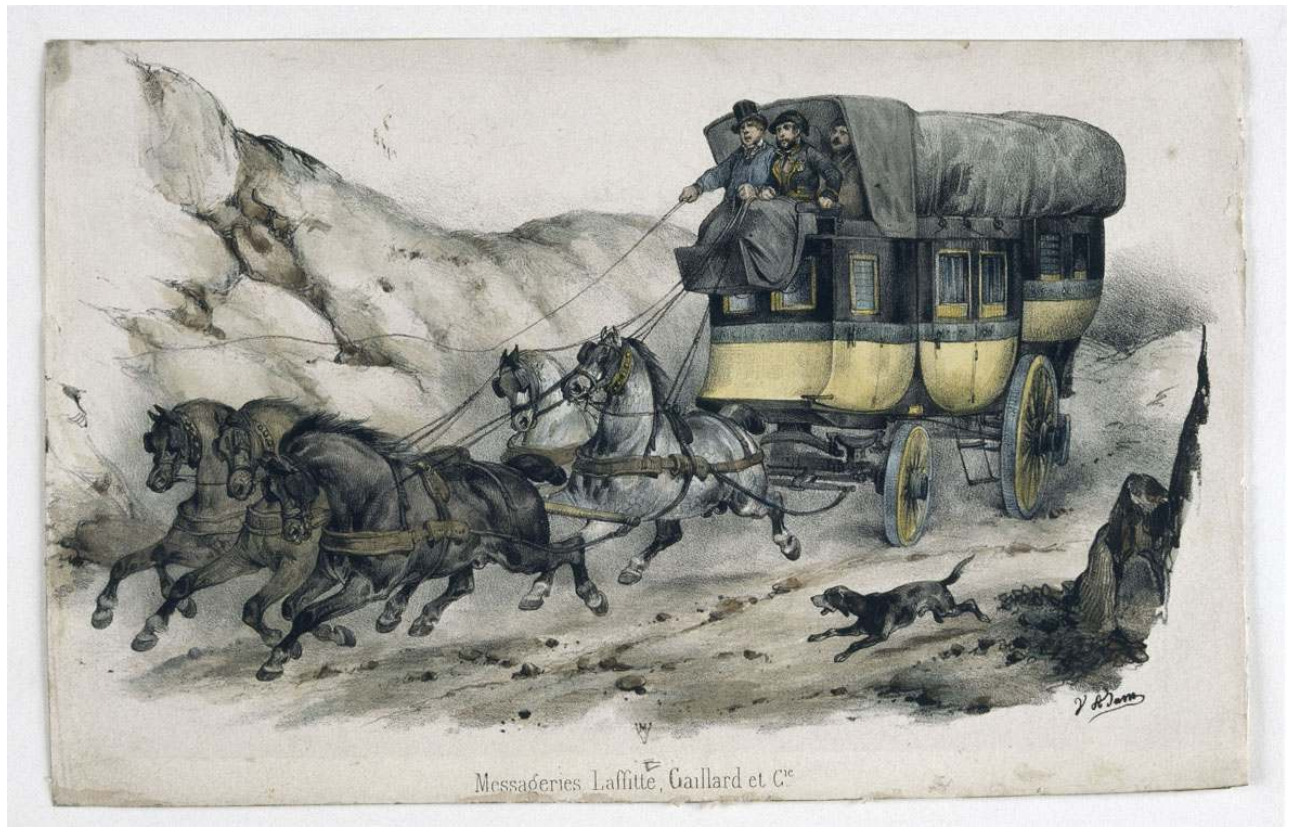

Adam, Victor-Jean (1801-1866). Messageries Laffitte, Caillard et Cie, lithographie, s. d., inv. CMV.2013.0.001.

Phot. Arnaudet, Daniel, RMN-Grand Palais (domaine de Compiègne). ( ) Agence photographique de la Réunion des Musées Nationaux : 96-008898.

L'attention de Victor-Jean Adam à la pénibilité du travail des chevaux anime une autre facette de son œuvre, que nous avons vue plus haut à propos des chevaux de coucous, et qui s'exprime dans une lithographie gravée par $C$. Motte représentant une diligence des Messageries royales (fig. 13). Il s'agit là aussi d'une diligence à trois compartiments de forme "classique", cette fois menée par un postillon. Le postillon et le conducteur ont tous deux mis pied à terre afin de soulager l'attelage à cinq chevaux qui tracte à grand peine la lourde voiture dans une côte, au pas. Cette attitude toute pragmatique témoigne d'une forme de respect à l'égard des chevaux, qui nous paraît pouvoir être étudiée en relation avec les obligations figurant dans la réglementation de la poste aux chevaux, qui visaient notamment à limiter l'usure des chevaux ${ }^{41}$. 


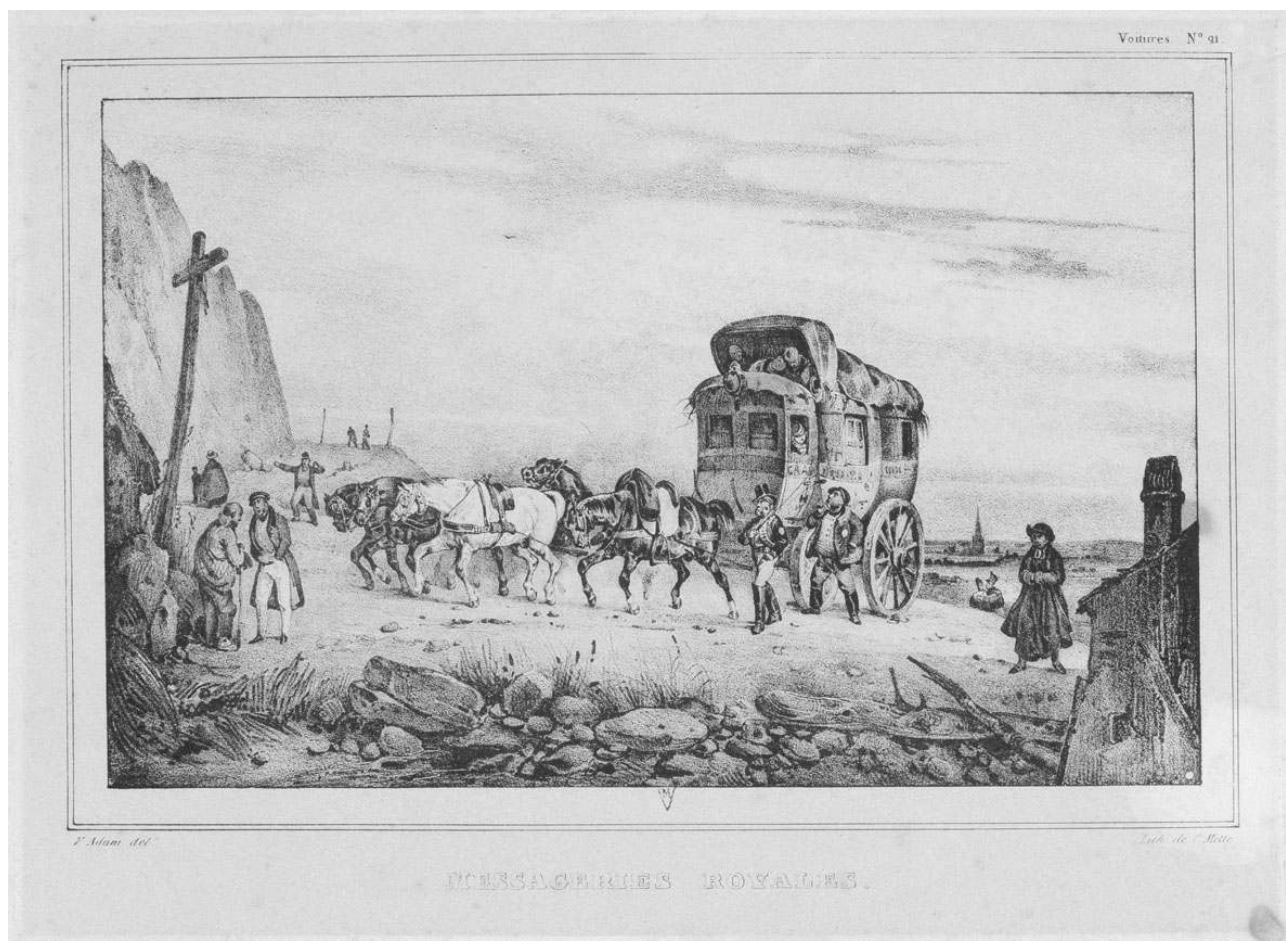

Motte, C. (lithographe), d'après Victor-Jean Adam (1801-1866). Messageries royales, Voitures n² 2 , lithographie, s.d. (vers 1830 ?), inv.CMV.883.

Phot. Arnaudet, Daniel, RMN-Grand Palais (domaine de Compiègne). @ Agence photographique de la Réunion des Musées Nationaux : 96-008701.

\section{La quotidienneté du travail des chevaux de malle-poste et de diligence}

Ce sont aussi les différentes étapes du voyage, qui sont autant de moments différenciés du travail des chevaux, que la collection du musée illustre. La lithographie d'A. Provost (actif de 1834 à 1855) intitulée Les messageries Laffitte et Caillard (CMV.1112) montre les instants précédant le départ d'une diligence dans les années 1840 . Pendant que les voyageurs font leurs adieux et prennent place dans la voiture, deux postillons règlent les derniers préparatifs, l'un attelant les chevaux tandis que l'autre semble vérifier la fixation de la capote ou des bagages sur le toit.

Halte de diligence, une peinture de Charles-Claude Delaye (1793-1848), datée vers 1830 (CMV.628), représente une diligence attelée de quatre chevaux, à l'arrêt devant un relais de poste de campagne, pour une des innombrables pauses imposées par le changement de chevaux. Ce moment est un thème qui semble avoir connu une faveur notable auprès des artistes, au regard de sa fréquence parmi les sujets traités au sein de la collection du musée.

51 Une lithographie de François Séraphin Delpech (1778-1825), d'après Horace Vernet (1789-1863), intitulée Malle-poste (fig. 14), datée vers 1818, campe cette opération cruciale pour le bon déroulement du trajet de ces voitures, qui étaient les plus rapides de leur temps. Ce changement se devait d'être accompli le plus rapidement possible afin de réduire au maximum la durée du trajet. Deux postillons, qui ont déchaussé leurs bottes 
pour plus de confort, s'affairent au dételage de l'attelage à quatre. L'un d'eux emmène les chevaux de tête vers l'écurie tandis que l'autre s'emploie à dételer les timoniers. Ce derniers sont saisis dans des attitudes de relâchement qui ne sont pas sans évoquer celles de coureurs, une fois la ligne d'arrivée franchie. À l'arrière-plan, un garçon d'écurie amène une paire de chevaux frais.

Figure 14

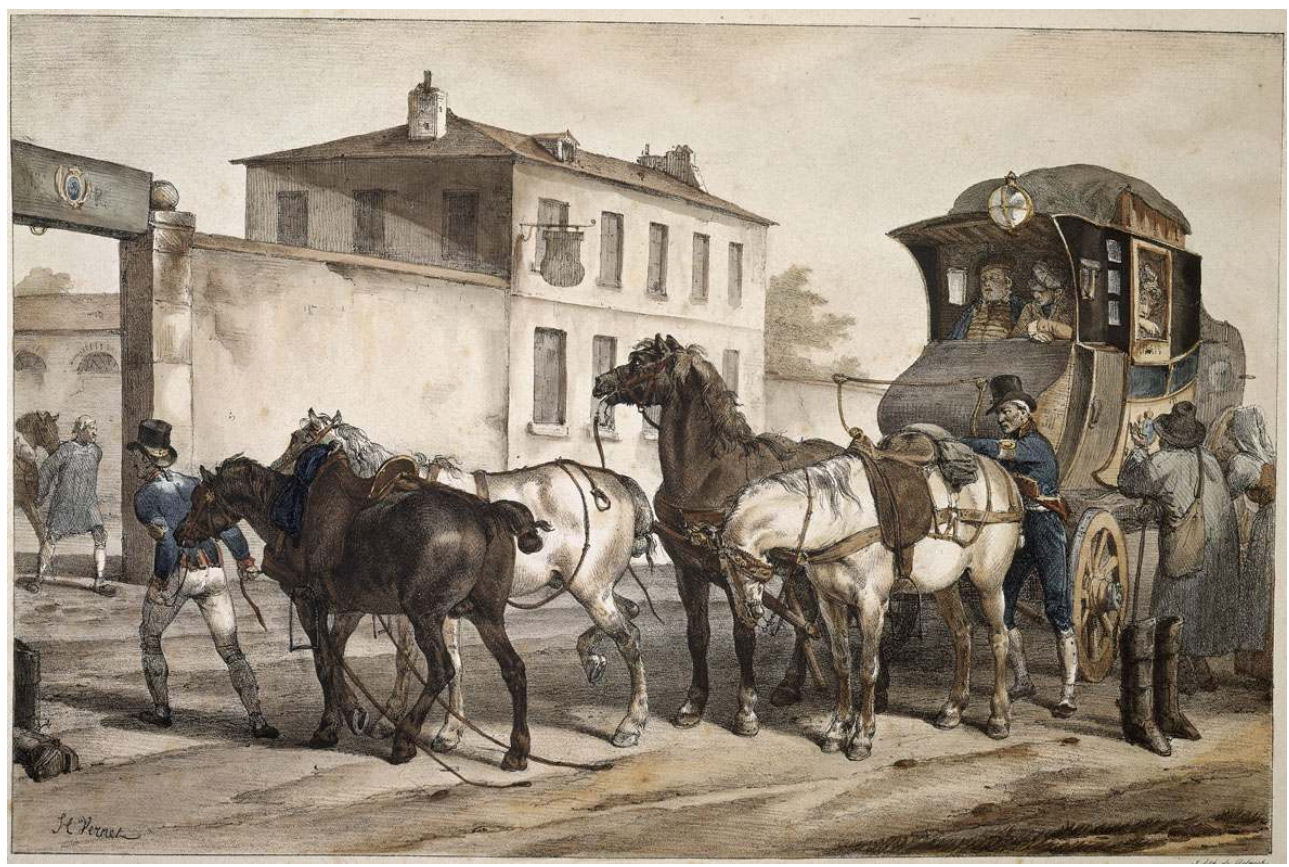

Delpech, François Séraphin (1778-1825), d'après Horace Vernet (1789-1863). Malle-poste, lithographie, s. d. (vers 1830 ?), inv.CMV.794.

Phot. RMN-Grand Palais (domaine de Compiègne) / Agence Bulloz. @ Agence photographique de la Réunion des Musées Nationaux : 01-023982.

\footnotetext{
Une autre lithographie, d'après A. Menut, lithographiée par Delaunois, intitulée Normandie et tirée de "L'Artiste» (CMV.972), représente une diligence à l'arrivée au relais, vers 1830, à ce même moment du dételage des chevaux. Les chevaux de volée ont déjà été dételés alors que les timoniers sont en train d'être détachés. Les signes de fatigue des chevaux y sont bien plus discrets, le rythme semble beaucoup plus lent, certains des voyageurs sont descendus de voiture, l'un d'eux s'étirant pour se dégourdir. Enfin, une peinture d'Auguste-Xavier Leprince (1799-1826), L'arrêt de la diligence à la poste royale (fig. 15), représente le même sujet, quelques minutes après. L'attelage est cette fois entièrement dételé. L'un des chevaux est montré s'éloignant seul de la voiture, tête basse, avec une lassitude mêlée de routine qui dispense les hommes de le guider.
} 


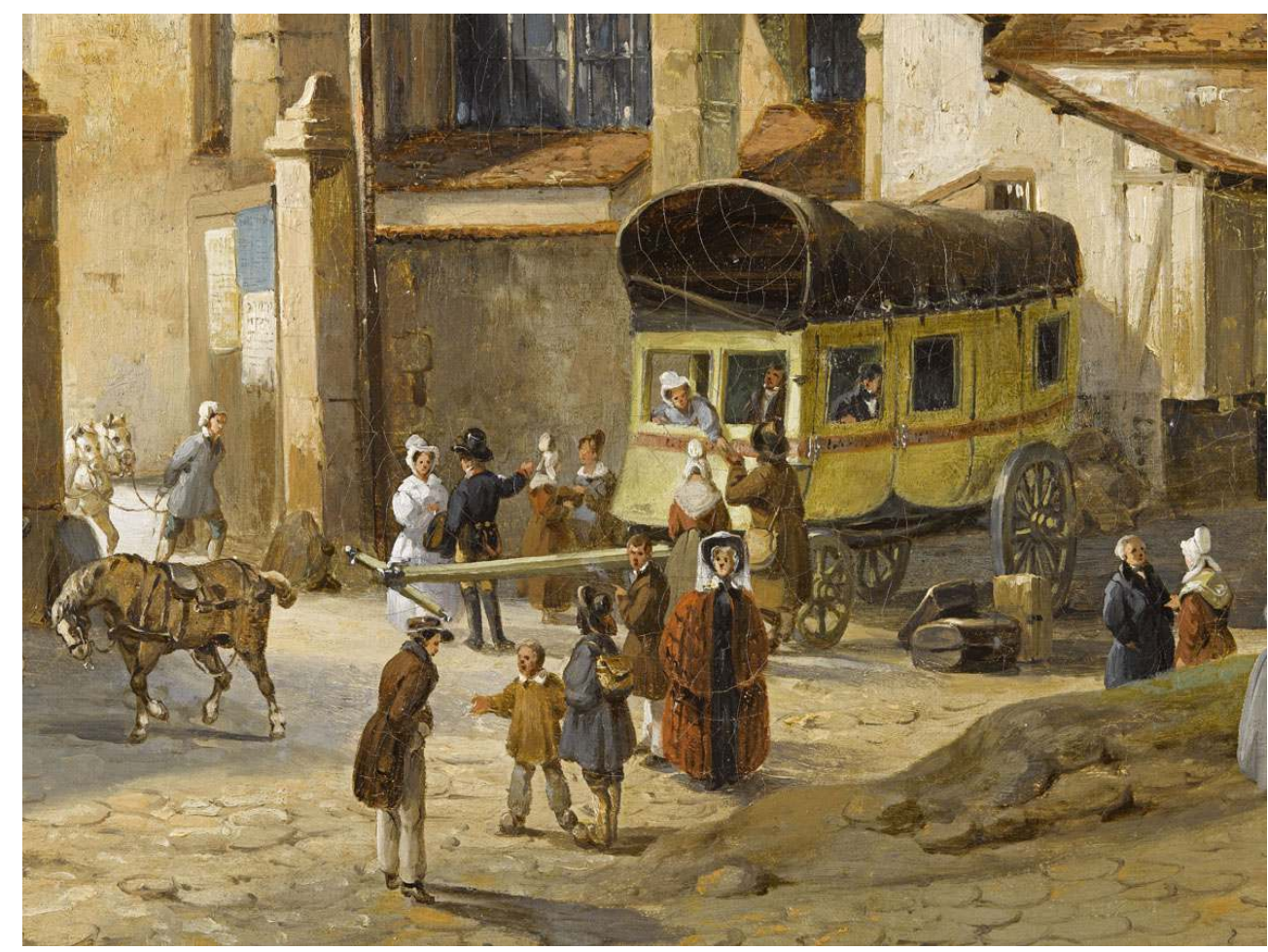

Leprince, Auguste-Xavier (1799-1826). L'arrêt de la diligence à la poste royale, détail, huile sur toile, vers 1820, inv. CMV.2005.002.

Phot. Ojéda, René-Gabriel, RMN-Grand Palais (domaine de Compiègne). (c) Agence photographique de la Réunion des Musées Nationaux : 08-518066.

\section{L'apparition de l'automobile et la recomposition de la place du cheval dans la société}

L'Apparition, une lithographie du peintre français William Laparra (1873-1920), datée 1906 (fig. 16), représente un de ces omnibus de campagne qui desservaient les routes secondaires à la fin $d u \mathrm{XIX}^{\mathrm{e}}$ siècle et au début du $\mathrm{Xx}^{\mathrm{e}}$ siècle. Les chevaux, éblouis par les puissants faisceaux des phares à acétylène d'une automobile surgissant dans la nuit, se sont arrêtés net, faisant voler l'ensemble des passagers. Si cette œuvre donne à voir l'une des raisons pour lesquelles ces techniques de transport étaient incompatibles, la frayeur des chevaux mettant directement en péril la vie des passagers de l'omnibus, elle peut aussi se lire comme une métaphore de la fin de l'ère de la traction animale, littéralement balayée par l'apparition de l'automobile. C'est une véritable mutation anthropologique, liée aux transformations des manières de se déplacer, que saisit l'artiste. 


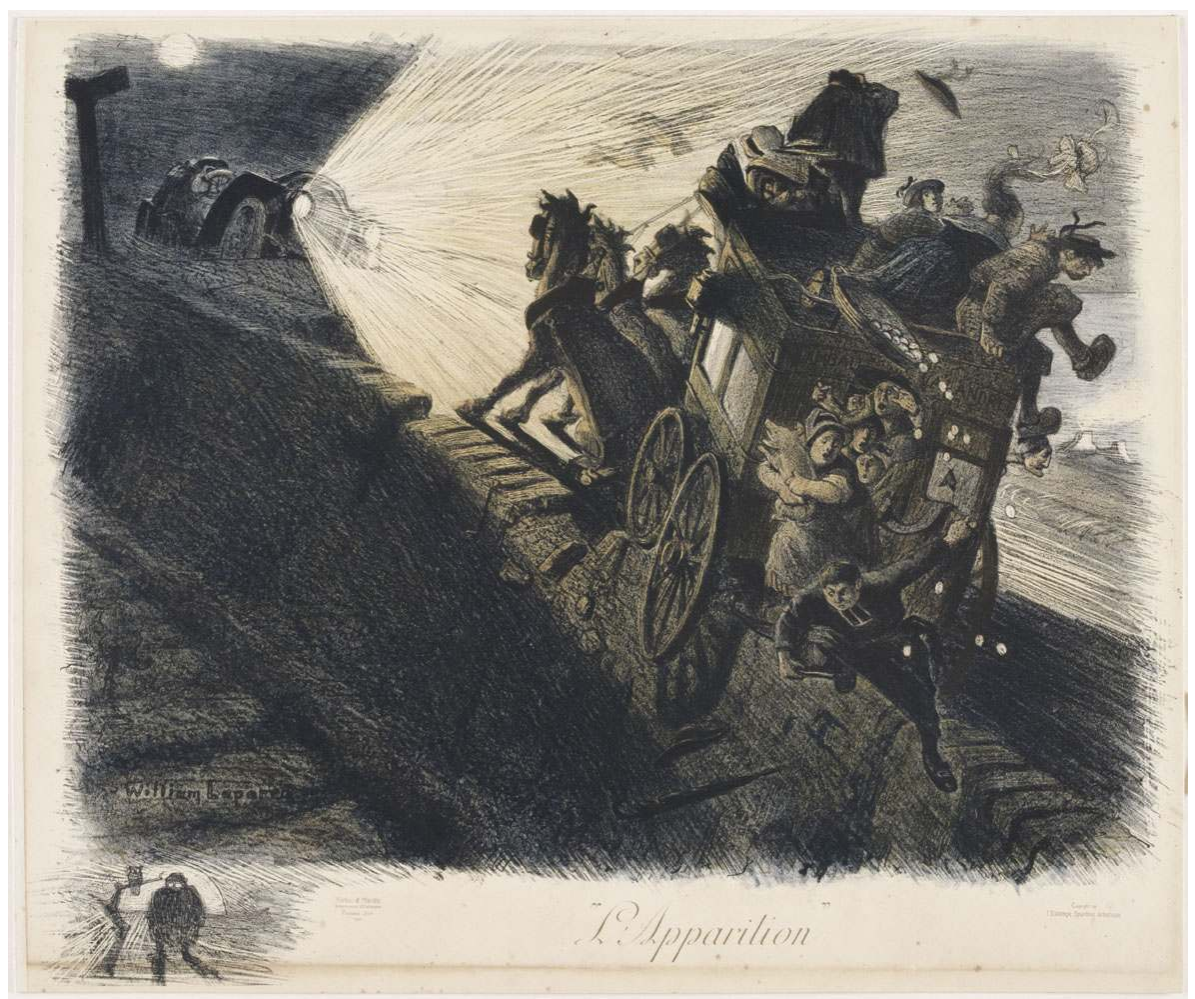

Laparra, William (1873-1920). L'Apparition, lithographie, 1906, inv. CMV.1258.

Phot. Blot, Gérard, RMN-Grand Palais (domaine de Compiègne). (c) Agence photographique de la Réunion des Musées Nationaux : 99-006017.

Pourtant, la traction animale ne disparut pas complètement. Une photographie récemment acquise, représentant une victoria de louage à l'arrêt près d'un bois, datée vers 1920 (fig. 17), témoigne de la persistance de l'usage de ces voitures hippomobiles en ville. L'attitude décontractée des passagers suggère que ce type de voiture faisait déjà l'objet d'une forme d'exploitation touristique. Ce phénomène nous paraît comparable au développement actuel de l'offre de promenades en voiture attelée dans les sites touristiques. Ainsi, le rôle du cheval, utilitaire dans l'industrie du transport, s'est-il mué en activité de plaisir dans le domaine des loisirs. 


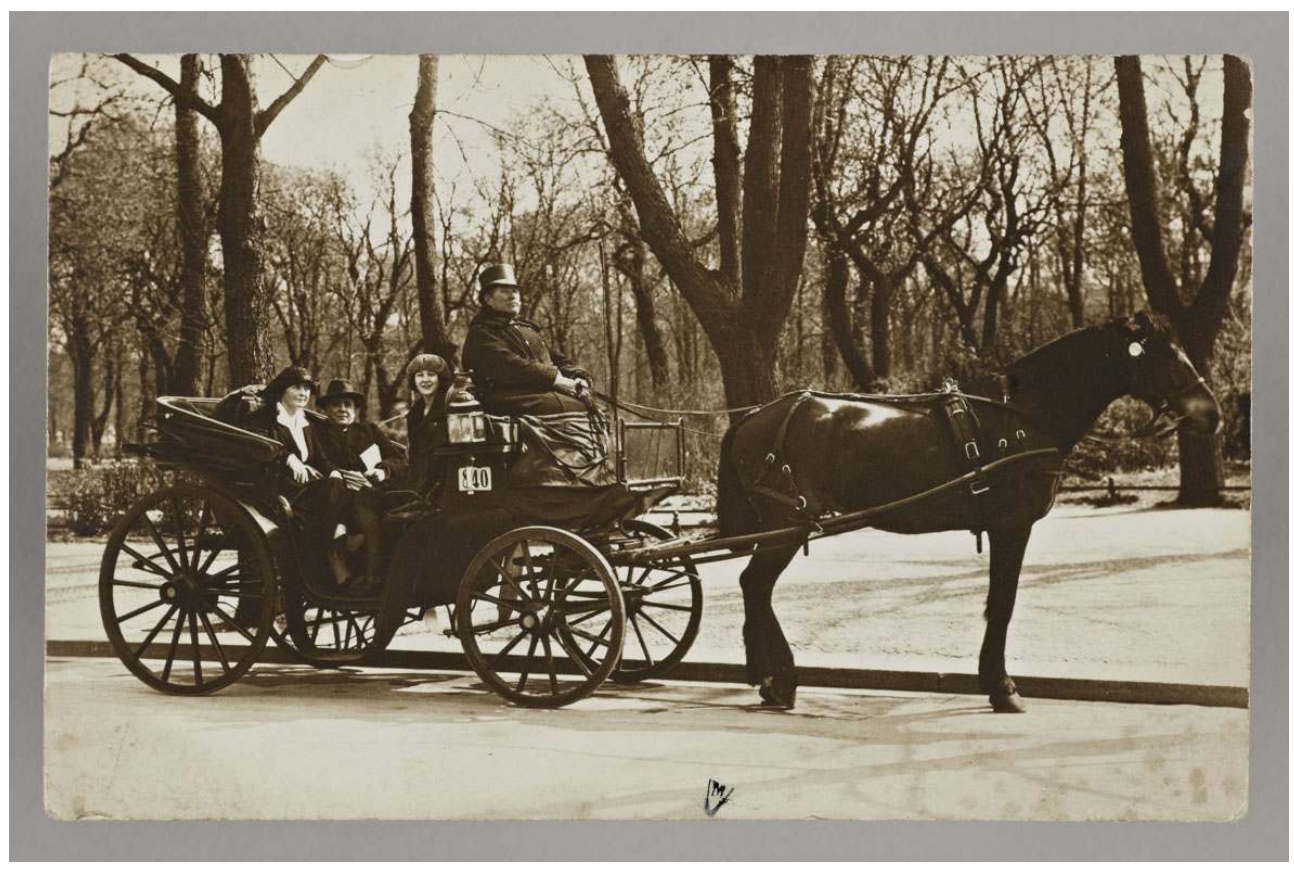

Anonyme. Une victoria de louage à l'arrêt auprès d'un bois, photographie, vers 1920, inv. CMV.2012.004. Phot. Maréchalle, Stéphane, RMN-Grand Palais (domaine de Compiègne). (c) Agence photographique de la Réunion des Musées Nationaux : 12-566294.

\section{Conclusion}

Les différents ensembles d'œuvres que nous avons sélectionnés peuvent être interprétés d'abord de façon chronologique, permettant de distinguer différents moments ou séquences dans l'histoire du transport public, à l'instar de la série qui illustre la naissance des omnibus parisiens ou encore des représentations de l'âge d'or des diligences. Les différents modes de transport participent d'un système dans lequel chaque modification d'une partie entraîne une adaptation des autres, à l'exemple du secteur des messageries, qui se recompose sous l'effet du développement des chemins de fer, puis disparait avec l'automobile. Les chevaux jouèrent tout au long du XIX siècle un rôle fondamental dans le développement de l'industrie du transport public, qui revêtait une importance économique et sociale considérable. La place des chevaux dans cette industrie évolua cependant, passant au fil du temps d'une position centrale à une position périphérique, d'une fonction pratique à celle d'animal de loisir.

C'est aussi la relation homme/animal, et à travers elle, l'évolution de la sensibilité à l'égard des chevaux de travail, liée à la naissance d'un mouvement de protection des animaux, que l'on peut observer dans ce corpus. Les représentations de chevaux maltraités ou défaillants sont plus nombreuses dans les œuvres relatives au transport public urbain, ce qui fait écho aux données rapportées par les différents auteurs consultés, qui soulignent le sort particulièrement cruel des chevaux des villes, sans toutefois écarter les difficultés du travail des chevaux dans le transport public routier. Aussi, à la lumière de ces données, des modes distincts de représentation des chevaux au travail se dessinent-ils au sein de ce corpus. Le regard des artistes traduit une vive 
compassion vis-à-vis des chevaux des villes exposés aux mauvais traitements et à leur tâche pénible, tandis que les représentations du transport public routier privilégient plutôt la beauté et la puissance des attelages, montrés en pleine course ou dans une routine besogneuse, comme lors du changement de chevaux au relais.

Nous espérons que cette sélection contribuera à mieux faire connaître les richesses de la collection du musée de la Voiture et du Tourisme, tant comme source iconographique pour la recherche scientifique que comme vaste ensemble d'œuvres, d'objets ou de documents pouvant être appréciés du public le plus large afin de prendre toute la mesure de la mutation anthropologique liée à la mécanisation et à la motorisation des transports.

\section{NOTES}

1. - L'auteure remercie Sandrine Grignon-Dumoulin pour sa contribution aux recherches sur les artistes.

2. - CHARBON, Paul. Au temps des malles-poste et des diligences. Histoire des transports publics et de poste du XVII $e^{e}$ au XIXe siècle. Schirmeck : Jean-Pierre Gyss, 1979, p. 8.

3. - Ibid.; MARCHAND, Patrick. Le maitre de poste et le messager. Les transports publics en France au temps des chevaux. Paris : Belin, 2006, p. 200-210, 230, 233, 239-240 ; NOUGARET, Pierre. " La Poste aux chevaux de 1740 à 1870 ». Dans Le voyage en France. Du maître de poste au chef de gare (1740-1914). Cat. exp. Compiègne, musée national de la Voiture et du Tourisme, avril-juillet 1997. Paris : RMN, 1997, p. 25-26.

4. - CHARBON, Paul. Op. cit., p. 12 ; JOBÉ, Joseph. Au temps des cochers. Histoire illustrée du voyage en voiture attelée $d u X^{e}$ au XXe siècle. Lausanne/Paris : Edita/Lazarus, 1976, p. 47.

5. - Les auteurs consultés divergent sur ce point, c'est pourquoi nous pensons que ces différentes configurations coexistaient au XIX ${ }^{\mathrm{e}}$ siècle. CHARBON, Paul. Op. cit., p. 7, 33 ; MARCHAND, Patrick. Op. cit., p. 43 ; TRÉNARD, Louis. «De la route royale à l'âge d'or des diligences ». Dans Les routes de France depuis les origines jusqu'à nos jours. Paris: Association pour la diffusion de la pensée française, 1959, p. 121.

6. - CHARBON, Paul. Op. cit., p. 7, 11-12; Id. «Les entreprises de messagerie, de Turgot au chemin de fer ». Dans Le voyage en France, Op. cit., p. 38-39; MARCHAND, Patrick. Op. cit., p. 30 ; STUDENY, Christophe. L'invention de la vitesse: France, XVIII ${ }^{e}-\mathrm{XX}^{e}$ siècle. Paris: Gallimard, 1995, p. 153; TRÉNARD, Louis. Op. cit., p. 121.

7. - CHARBON, Paul. Op. cit., p. 10 ; MARCHAND, Patrick. Op. cit., p. 20-21, 33, 37, 43 ; TRÉNARD, Louis. art. cit., p. 120-121.

8. - BOUCHET, Ghislaine. Le cheval à Paris de 1850 à 1914. Genève : Droz, 1993, p. 83 ; LAGARRIGUE, Louis. Cent ans de transports en commun dans la région parisienne. T. I. Organisation administrative et exploitation. Paris: Régie autonome des Transports parisiens, 1956, p. 22-23; PAPAYANIS, Nicholas. Horse-drawn Cabs and Omnibuses. The Idea of Circulation and the Business of Public Transit. Baton Rouge/Londres : Louisiana State University Press, 1996, p. 13-14.

9. - BOUCHET, Ghislaine. Op. cit., ibid. ; DIGARD, Jean-Pierre. Une histoire du cheval. Art, techniques, société. Arles : Actes Sud, 2004, p. 134 ; LAGARRIGUE, Louis. Op. cit., p. 27 ; PAPAYANIS, Nicholas. Op. cit., p. 16-21.

10. - Ibid., p. 14-15 ; LAGARRIGUE, Louis. Op. cit., p. 27-28, 198.

11. - DIGARD, Jean-Pierre. Op. cit., p. 135. 
12. - LEROI-GOURHAN, André. « Préface ». Dans LIZET, Bernadette. Le cheval dans la vie quotidienne. Techniques et représentations du cheval de travail dans l'Europe industrielle. Paris : Jean-Michel Place, 1996 ( $1^{\mathrm{e}}$ édition : 1982).

13. - BOUCHET, Ghislaine. Op. cit., p. 83-107 ; CHARBON, Paul. Op. cit., p. 10, 39 ; DIGARD, JeanPierre. Op. cit., p. 153.

14. - MARCHAND, Patrick. Op. cit., p. 64-65 ; TRÉNARD, Louis. art. cit., p. 120-121.

15. - Nous avons principalement retenu ces désignations pour plus de simplicité, bien que ces deux entreprises aient connu plusieurs noms et soient donc désignées de différentes façons suivant les auteurs. Fondée sous le nom d'«Entreprise générale des messageries », la première est aussi connue sous le nom d'«Entreprise générale ", de "Messageries impériales " sous le Premier et le Second Empire, et de «Messageries royales», qui est la désignation la plus fréquente. Pour la seconde, on relève les désignations suivantes : "Entreprise des messageries générales de France, Laffitte et Caillard et cie», "Messageries générales ", "messageries générales Laffitte et Caillard », " messageries Laffitte et Caillard », "Laffitte et Caillard et cie » et «Laffitte et Caillard», cette dernière étant la plus courante. CHARBON, Paul. Op. cit, p. 28-33; id. «Les entreprises de messagerie", art. cit., p. 40-45; MARCHAND, Patrick. Op. cit., p. 57-60 ; PAPAYANIS, Nicholas. Op. cit., p. 135 ; STUDENY, Christophe. Op. cit., p. 149 ; TRÉNARD, Louis. art. cit., p. 125.

16. - MARCHAND, Patrick. Op. cit., p. 71-72.

17. - CHARBON, Paul. Op. cit., p. 28-30, 33 ; id. « Les entreprises de messagerie », art. cit., p. 41-44 ; MARCHAND, Patrick. Op. cit., p. 49 ; TRÉNARD, Louis. art. cit., p. 123-124.

18. - CHARBON, Paul. Op. cit., p. 33 ; id. «Les entreprises de messagerie », art. cit., p. 42-44 ; STUDENY, Christophe. Op. cit., p. 149-156.

19. - CHARBON, Paul. Op. cit., p. 53-60 ; DIGARD, Jean-Pierre. Op. cit., p. 132 ; MARCHAND, Patrick. Op. cit., p. 130-131.

20. - CHARBON, Paul. Op. cit., p. 10.

21. - Ibid. p. 38, 59-60; id. « Les entreprises de messagerie », art. cit., p. 45.

22. - LAGARRIGUE, Louis. Op. cit., p. 30, 56 ; PAPAYANIS, Nicholas. Op. cit., p. 6-7 ; STUDENY, Christophe. Op. cit., p. 116-117.

23. - BOUCHET, Ghislaine. Op. cit., p. 85 ; DEVAUGES, Jean-Denys. Notice d'acquisition ( $\left.{ }^{\circ} 43\right)$ d'un modèle réduit de fiacre de la compagnie parisienne "L'Urbaine ». La revue des musées de France. Revue du Louvre, 2005, nº 3, p. 100-101 ; PAPAYANIS, Nicholas. Op. cit., p. 137-140, 149-153.

24. - BOUCHET, Ghislaine. Op. cit., p. 53.

25. - Ibid. p. 84-85; STUDENY, Christophe. Op. cit., p.116; LIBOUREL, Jean-Louis. Voitures hippomobiles. Vocabulaire typologique et technique. Paris : Éditions du patrimoine, 2005, p. 70-127.

26. - LAGARRIGUE, Louis. Op. cit., p. 27.

27. - LAGARRIGUE, Louis. Op. cit., p. 28 ; PAPAYANIS, Nicholas. Op. cit., p. 57-58, 60-61; RUMIN, Marcel. «Les omnibus nantais ». L'histoire des transports à Nantes. Les Annales de Nantes et du Pays nantais. Revue de la Société académique de Nantes et de la Loire-Atlantique, 2004, n 294, p. 1.

28. - LAGARRIGUE, Louis. Op. cit., p. 28-31 ; PAPAYANIS, Nicholas. Op. cit., p. 60-63.

29. - BOUCHET, Ghislaine. Op. cit., p. 94 ; LIZET, Bernadette. Op. cit., p. 111 ; STUDENY, Christophe. Op. cit., p. 117.

30. - La datation retenue est celle qui est donnée par Françoise Reynaud. Il est à noter toutefois que Nicholas Papayanis date cette prise de vue de 1898 dans l'ouvrage précédemment cité, cahier d'illustrations, non paginé. REYNAUD, Françoise. Les voitures d'Atget au musée Carnavalet. Paris : Carré/Paris Musées, 1991, p. 5-12.

31. - LAGARRIGUE, Louis. Op. cit., p. 103 et 118.

32. - BOUCHET, Ghislaine. Op. cit., p. 53, 87 ; PAPAYANIS, Nicholas. Op. cit., p. 77.

33. - BENJAMIN, Walter. L'œuvre d'art à l'époque de sa reproductibilité technique. Paris : Éditions Allia, 2011, p. 32 ; LIZET, Bernadette. Op. cit., p. 161 ; REYNAUD, Françoise.Op. cit., p. 8. 
34. - BOUCHET, Ghislaine. Op. cit., p.181-182 ; DIGARD, Jean-Pierre. Op. cit., p. 178 ; LIZET, Bernadette. Op. cit., p. 123-127.

35. - BOUCHET, Ghislaine. Op. cit., p. 86, p. 94 ; LIZET, Bernadette. Op. cit., p. 111-113 ; MOM, Gjis. The Electric Vehicle. Technology and Expectations in the Automobile Age. Baltimore: The Johns Hopkins University Press, 2004, p. 71.

36. - LIZET, Bernadette. Op. cit., p. 123-124.

37. - BOUCHET, Ghislaine. Op. cit., p. 176-177 ; LIZET, Bernadette. Op. cit., p. 123-124 ; PAPAYANIS, Nicholas. Op. cit., p. 159.

38. - CHARBON, Paul. Op. cit., p. 39 et 60 ; STUDENY, Christophe. Op. cit., p. 153.

39. - CHARBON, Paul. Op. cit., p. 102 ; TRÉNARD, Louis. Op. cit., p. 123.

40. - CHARBON, Paul. Op. cit., p. 105 ; TRÉNARD, Louis. Op. cit., p. 123.

41. - CHARBON, Paul. Op. cit., p. 78.

\section{RÉSUMÉS}

À partir d'une sélection d'œuvres provenant du fonds d'arts graphiques et de photographies ainsi que de la collection de peintures du musée de la Voiture et du Tourisme de Compiègne, cette communication s'intéresse aux différents modes ou régimes de représentation du cheval au travail dans le transport public français, du XIX siècle, période qui marque l'apogée d'un système de transports fondé sur le cheval, au début $\mathrm{du} \mathrm{XX}^{\mathrm{e}}$ siècle, où l'on observe une remise en question de la place du cheval dans la société, sous l'effet du développement de l'automobile. Le transport public urbain et le transport public routier y sont successivement explorés. Au-delà de la présentation des spécificités des principaux types de voitures publiques qui caractérisaient cette période, cette sélection met en lumière l'évolution de la sensibilité à l'égard des chevaux de travail, liée à la naissance d'un mouvement de protection des animaux. Les représentations montrant la maltraitance et les difficultés du travail des chevaux apparaissent plus prégnantes dans le domaine du transport public urbain, tandis que les représentations du transport public routier privilégient plutôt la beauté et la puissance des attelages, montrés en pleine course ou sous l'espèce de la quotidienneté de leur travail.

The horse in nineteenth-century public transport, in the collections of the national carriage and tourism museum at Compiègne. Based on a selection of the paintings, drawings and photographs held by the Compiègne museum, this paper will look at the different ways the horse was depicted in its contribution to nineteenth-century public transport. This century marked the apogee of the use of the horse, brought into question from the beginning of the twentieth century by the appearance of the motor car. Urban and inter-city public systems will be looked at in turn. Beyond a presentation of the specificities of certain horse-drawn vehicles characteristic of the period, this selection of graphic representations also throws light on society's evolving attitudes towards these workhorses, related to the birth of a movement for the protection of animals. The pictures showing mistreatment of horses or the difficulties of their work seem to be more numerous where urban transport modes are concerned. The images of inter-city transport tens to draw more attention to the beauty and the strength of the horse teams, often shown at full gallop, rather than in some everyday position. 


\section{INDEX}

Mots-clés : cheval de travail, transport public, transport en commun, XIXe siècle, voiture hippomobile, représentation, attelage, regard d'artiste, voiture de louage, fiacre, coucou, omnibus, diligence, malle-poste, relation homme-animal, protection des animaux, mutation anthropologique, tourisme

Keywords : workhorse, public transport, nineteenth century, horse-drawn vehicle, artist's viewpoint, hired carriage, hackney cab, stagecoach, protection of animals, tourism

\section{AUTEUR}

\section{ÉLISE FAU}

conservatrice du patrimoine, musée national de la Voiture et du Tourisme, Compiègne elise.fau@culture.gouv.fr 